\title{
Anomalías neurocognitivas asociadas al consumo intensivo de alcohol (binge drinking) en jóvenes y adolescentes: Una revisión
}

\author{
Neurocognitive anomalies associated with the \\ binge drinking pattern of alcohol consumption in \\ adolescents and young people: A review
}

\author{
Eduardo lópez-Caneda*, Nayara Mota**, Alberto Crego***, Teresa Velasquez***, Montserrat \\ Corral*, Socorro Rodríguez Holguín*, Fernando Cadaveira* \\ * Departamento de Psicoloxía Clínica e Psicobioloxía, Universidade de Santiago de Compostela, Galicia, España. \\ ** Departamento de Fundamentos de Psicologia, Universidade do Estado do Rio de Janeiro, Brasil. \\ *** Neuropsychophysiology Lab, Centro de Investigação em Psicologia (CIPsi), Escola de Psicologia, Universidade do Minho, \\ Braga, Portugal.
}

\section{Resumen}

El consumo intensivo de alcohol o binge drinking (BD) constituye la forma de consumo problemático más común durante la adolescencia y juventud. Al mismo tiempo, es éste un período marcado por profundos cambios, tanto en la estructura como en el funcionamiento cerebral, que pueden verse afectados por el consumo intensivo de alcohol. En los últimos años, se ha publicado un importante número de estudios que tratan de caracterizar los efectos del BD sobre el cerebro. Sin embargo, no existe hasta la fecha ninguna revisión crítica en lengua española de la investigación sobre las consecuencias neuroestructurales, neurofuncionales y cognitivas que pueden derivarse del mantenimiento de un patrón de consumo intensivo de alcohol durante la adolescencia y juventud. El propósito de esta revisión es hacer un resumen crítico de los principales resultados de la investigación sobre los efectos del BD en el cerebro. Para ello, se realizó una búsqueda bibliográfica en las bases de datos Web of Knowledge, PubMed y PsycINFO para el periodo 2000-2013. En general, los trabajos coinciden en señalar que el $\mathrm{BD}$ se asocia a 1) menor rendimiento en tareas que evalúan procesos cognitivos como la atención, la memoria o las funciones ejecutivas, 2) alteraciones estructurales (en sustancia blanca y en sustancia gris) en distintas regiones cerebrales y 3) anomalías neurofuncionales (hiper- y/o hipoactivación neural) ligadas a distintos procesos cognitivos. Estos resultados, si bien aún necesitan ser contrastados, alertan sobre las importantes consecuencias que podría tener la persistencia del BD sobre un cerebro joven y todavía en maduración.

Palabras Clave: alcohol, binge drinking, adolescencia neuropsicología, neuroimagen.

\begin{abstract}
Binge drinking (BD) is the most common problematic drinking pattern during adolescence and youth. At the same time, it is a period marked by profound structural and functional brain changes, which may be affected by heavy alcohol consumption. In recent years, a considerable number of studies that attempt to characterize the effects of $\mathrm{BD}$ on the brain has been published. However, to date there is not any critical review in Spanish language on neurostructural, neurophysiological and cognitive consequences that may result from the maintenance of a BD pattern of alcohol consumption during adolescence and youth. The purpose of this review is to critically summarize the main research results on the effects of $\mathrm{BD}$ on the brain. To this end, a literature search in databases Web of Knowledge, PubMed and PsycINFO for the period 2000-2013 was performed. In general, studies agree that $\mathrm{BD}$ is associated with 1) lower performance on tasks assessing cognitive processes such as attention, memory and executive functions, 2) structural changes (in white matter and gray matter) in different brain regions and 3) neurophysiological abnormalities (hyper/hypoactivation) linked to different cognitive processes. These results, although still need to be contrasted, warn about important consequences that could result from the persistence of $\mathrm{BD}$ on a young and still maturing brain.
\end{abstract}

Key Words: alcohol, binge drinking, adolescence, neuropsychology, neuroimaging 
$\mathrm{E}$ n las últimas décadas, ha adquirido un creciente protagonismo social y sanitario un patrón de consumo caracterizado por la ingesta de grandes cantidades de alcohol en un corto espacio de tiempo, que lleva frecuentemente a la embriaguez, y se alterna con períodos de abstinencia entre los episodios de consumo intensivo (Courtney y Polich, 2009; Parada et al, 2011a).

La importancia que el binge drinking (BD) -como se conoce en la literatura anglosajona a este patrón de consumo- ha adquirido en los últimos años viene dada, fundamentalmente, por las significativas consecuencias sociosanitarias asociadas al mismo (accidentes de tráfico, agresiones, bajo rendimiento académico, alteraciones cardiovasculares, etc.) (Goslawski et al., 2013; Mota et al., 2010; Svensson y Landberg, 2013; Valencia-Martín, Galán y Rodríguez Artalejo, 2008), así como por la alta prevalencia que presenta entre los jóvenes y adolescentes en la mayoría de los países occidentales (Hibell et al., 2009; Observatorio Europeo de las Drogas y las Toxicomanías, OEDT, 2011; 2012; Substance Abuse and Mental Health Services Administration, SAMHSA, 2013). Sin embargo, a pesar del creciente número de estudios al respecto, el conocimiento sobre las consecuencias neurocognitivas de esta forma de consumo de alcohol es escaso y la concienciación social ante esta problemática es todavía reducida.

Si bien en el último año han aparecido algunas revisiones en inglés sobre esta temática (Jacobus y Tapert, 2013; Petit, Maurage, Kornreich, Verbanck y Campanella, 2014), hasta la fecha no se ha realizado una revisión crítica en lengua española de los estudios sobre los efectos del BD en el cerebro de los jóvenes y adolescentes sin trastornos de abuso o dependencia del alcohol. El objetivo de la presente revisión es cubrir ese vacío y acercar los conocimientos adquiridos a lo largo de la última década, además de a los profesionales del campo de las adicciones, a los profesionales de la salud y la educación que trabajan con esta población subclínica de consumidores intensivos de alcohol. Para contextualizar el marco de los estudios en este campo, en esta introducción se hace un breve repaso a la definición y epidemiología del BD y a las posibles causas de la especial vulnerabilidad del cerebro adolescente a los efectos del alcohol. A continuación, se presentará la revisión y discusión de los estudios que han explorado la posible presencia de anomalías, tanto a nivel cognitivo como neuroestructural y neurofuncional (lo que en este trabajo se engloba bajo el término de anomalías neurocognitivas), en los jóvenes y adolescentes binge drinkers (BDs).

\section{Definición del consumo intensivo de alcohol o binge drinking}

En los años 90, a partir de una investigación de la Harvard School of Public Health College Alcohol Study (Wechsler, Davenport, Dowdall, Moeykens y Castillo, 1994), se definió el BD como el consumo de cinco o más bebidas alcohólicas en varones y cuatro o más en mujeres, en una única ocasión, al menos una vez en las últimas dos semanas. Sin embargo, aunque actualmente la definición de Wechsler y cols. está bastante extendida, existe cierta controversia en torno a ella.

Principalmente, se ha planteado la necesidad de tener en cuenta la concentración de alcohol en sangre (CAS) para determinar de forma más precisa cuál es el umbral adecuado para establecer un patrón BD (Beirness, Foss y Vogel-Sprott, 2004; Lange et al., 2002; Naimi y Brewer, 2005). Por este motivo, el National Institute on Alcohol Abuse and Alcoholism (NIAAA) redefinió en 2004 el término BD como un consumo de alcohol que eleva la CAS a 0,08 g/dL. En adultos, esto equivale a 5/4 (hombres/mujeres) o más bebidas alcohólicas en aproximadamente 2 horas (NIAAA, 2004). Sin embargo, dado que los gramos de alcohol por unidad de bebida varían considerablemente de unos países a otros, es necesario adaptar el número de bebidas requeridas para alcanzar el umbral BD al país en el que se desarrolle el estudio. Así, mientras en Estados Unidos una unidad de bebida estándar (UBE) equivale a $14 \mathrm{~g}$ de etanol, en buena parte de los países europeos (entre los que se encuentra España) una UBE equivale a $10 \mathrm{~g}$. De este modo, en estos países una aproximación más adecuada a los criterios de la NIAAA sería el de 6 o más UBEs (60 g) para hombres y 5 o más ( 50 g) para mujeres en un intervalo aproximado de 2 horas, consumo que resulta, aproximadamente, en una CAS de 0,08 g/dL.

Otra variable de relevancia para definir de forma más completa y precisa el patrón $\mathrm{BD}$ es la frecuencia con la que se producen los episodios de consumo intensivo. Aunque no existe un criterio uniforme para caracterizar el patrón BD, lo más extendido y aceptado actualmente es considerar que los episodios de consumo intensivo deben producirse al menos una vez al mes o una vez cada dos semanas (Courtney y Polich, 2009; Parada et al., 2011a).

En definitiva, a la hora de estudiar el patrón BD se hace necesario tener en cuenta diversos aspectos como la cantidad, la rapidez o la frecuencia de consumo de alcohol. La combinación de estas variables, así como la adaptación de las mismas al país dónde se realice el estudio, dificulta sumamente el establecimiento de una definición operativa unánime del BD. En España, el Observatorio Español de la Droga y las Toxicomanías (OEDT) lo define como el consumo en la misma ocasión de 5 o más bebidas alcohólicas para los hombres y 4 o más en el caso de las mujeres, durante los últimos 30 días (OEDT, 2012). A nivel internacional, la que actualmente está más aceptada es el consumo de 5/4 o más unidades de bebida alcohólica, en un intervalo de aproximadamente dos horas, al menos una vez al mes.

\section{Epidemiología}

Tanto en Europa como en Norteamérica, la proporción de BD es mayor en los grupos de edad más jóvenes -entre 15 y 24 años- y en los estudiantes que en el resto de grupos de edad y ocupación, siendo mayor entre los hombres que 
entre las mujeres (Eurobarometer, 2010; SAMHSA, 2013). España ocupa el quinto lugar entre los países europeos en número de BDs (34\% de los jóvenes entre 15 y 24 años), cinco puntos por encima de la media de la Unión Europea (29\%) (Eurobarometer, 2010). Según los últimos informes del OEDT, el grupo de edad que presenta mayor prevalencia de BD es el de 20 a 24 años, siendo mayor entre los hombres (35\%) que entre las mujeres (20\%) (OEDT, 2012). En los menores de edad, en torno al 35-40\% de los estudiantes de 14 a 18 años reconocieron haber practicado el $\mathrm{BD}$ en el último mes ( $51 \%$ de los jóvenes de 17 años) (OEDT, 2013).

\section{El cerebro adolescente: un cerebro en desarrollo, un cerebro vulnerable}

La adolescencia es un período de profundos cambios, caracterizados por una amplia variedad de transformaciones a nivel emocional, cognitivo y conductual (Dahl, 2004; Spear, 2000). Buena parte de estos cambios están sujetos a los procesos madurativos que tienen lugar en el cerebro. Aunque la mayor parte del desarrollo cerebral tiene lugar antes de los cinco años (Dubois et al., 2008; Hermoye et al., 2006), la neuromaduración humana, lejos de finalizar en la infancia, se extiende a lo largo de la adolescencia y el inicio de la etapa adulta (Blakemore, 2012; Østby et al., 2009; Shaw et al., 2008).

A través primero de los estudios post-mortem (Huttenlocher, De Courten, Garey y Van der Loos, 1983; Yakovlev y Lecours, 1967) y más tarde de los estudios de imagen por resonancia magnética (IRM) (Giedd et al., 1996, 1999; Gogtay et al., 2004), se ha observado reiteradamente que el cerebro -particularmente las cortezas de asociación de orden superior, tales como el córtex prefrontal (CPF), el córtex parietal posterior o el córtex temporal inferior- experimenta fundamentalmente dos tipos de cambios a lo largo de la adolescencia.

El primero de ellos tiene que ver con la reorganización sináptica, esto es, la poda o eliminación de ciertas conexiones escasamente usadas y el fortalecimiento o potenciación de aquellas empleadas con frecuencia. Se cree que este proceso permite una mejora en la comunicación entre las redes neurales, haciendo a los circuitos sinápticos más eficientes (Casey, Galvan y Hare, 2005). Mientras que la poda sináptica en regiones sensoriales y motoras tiene lugar relativamente temprano durante el curso del desarrollo, otras regiones corticales de orden superior presentan un desarrollo más tardío (Fuster, 2002). Es el caso del CPF, cuyos cambios en la densidad sináptica (el número de sinapsis por unidad de volumen cerebral) parecen seguir un patrón de U invertida, con un incremento en la proliferación de sinapsis al comienzo de la adolescencia seguido de un decremento gradual de la densidad sináptica que se extiende hasta el comienzo de la edad adulta (Giedd et al., 2009; Gogtay et al., 2004).
Junto a los cambios que tienen lugar en la sustancia gris, el otro gran proceso neuromadurativo que tiene lugar durante la adolescencia y el inicio de la etapa adulta es la mielinización. La formación de las vainas de mielina que envuelven a los axones conlleva un incremento en la velocidad de conducción de los potenciales de acción y, consecuentemente, en la velocidad de transmisión de la información neural. Al igual que en los procesos de reorganización sináptica, las regiones que finalizan más tarde el proceso de mielinización son las áreas asociativas de orden superior (Fuster, 2001). Diversos estudios han mostrado que este proceso sigue un curso lineal, incrementándose a lo largo del desarrollo (Giedd, 2004), y que ciertas conexiones o tractos de sustancia blanca, como las fibras de asociación del CPF, presentan aumentos en el volumen de sustancia blanca incluso después de los 30 años (Lebel y Beaulieu, 2011).

Dada la estrecha relación entre desarrollo cognitivo y maduración cerebral, la reorganización sináptica y la mielinización modulan el desarrollo de los procesos cognitivos. Así, en numerosos estudios se ha observado como, a medida que el CPF y otras áreas de orden superior se desarrollan, funciones cognitivas como el control inhibitorio, la memoria de trabajo o la toma de decisiones experimentan mejoras significativas (Hooper, Luciana, Conklin y Yarger, 2004; Luna y Sweeney, 2004; Tamm, Menon y Reiss, 2002). La relativa inmadurez de estos procesos cognitivos en la adolescencia puede explicar, al menos en parte, la mayor propensión a involucrarse en conductas de riesgo tales como el consumo de sustancias en esta edad (Bava y Tapert, 2010; López-Caneda, Rodríguez Holguín, Cadaveira, Corral y Doallo, 2014). En este contexto de desarrollo de la estructura y funcionamiento cerebral, el consumo de alcohol puede ejercer un poderoso impacto negativo sobre el proceso neuromadurativo y, en consecuencia, alterar el funcionamiento normal de procesos cognitivos que son esenciales para una correcta adaptación a la vida adulta.

\section{Vulnerabilidad del cerebro adolescente a los efectos del alcohol: evidencia desde los estudios animales}

Dado que el consumo de alcohol durante la adolescencia puede perturbar el curso del desarrollo de ciertas regiones cerebrales, no es de extrañar que cantidades similares de alcohol puedan conllevar consecuencias diferentes en los adolescentes que en los adultos. En este sentido, en la última década diversos estudios con animales han mostrado que, efectivamente, el cerebro adolescente resulta ser particularmente sensible a los efectos perjudiciales del alcohol. Así, se ha observado que las ratas adolescentes expuestas a un patrón BD presentan mayor afectación estructural, especialmente en regiones como el CPF o el hipocampo, que ratas adultas expuestas a niveles equivalentes de alcohol (Crews, Braun, Hoplight, Switzer y 
Knapp, 2000; Pascual, Pla, Miñarro y Guerri, 2014; White y Swartzwelder, 2004). Del mismo modo, las ratas adolescentes con un patrón BD presentan peor rendimiento que las adultas en tareas que implican aprendizaje y memoria (Markwiese, Acheson, Levin, Wilson y Swartzwelder, 1998; Risher et al., 2013; Sircar y Sircar, 2005), lo cual parece ser indicativo de una mayor afectación neurocognitiva en las ratas más jóvenes.

En resumen, 1) la adolescencia es un período marcado por profundos cambios en el cerebro necesarios para el funcionamiento apropiado de funciones cognitivas tales como la memoria de trabajo o el control inhibitorio (Casey, Giedd y Thomas, 2000; Luna y Sweeney, 2004); 2) el alcohol es la droga más comúnmente usada entre los jóvenes y adolescentes (Eurobarometer, 2010; Hibell et al., 2009); 3) el BD es la forma de consumo problemático más común a esas edades (Farke y Anderson, 2007; Krauss, Baumeister, Pabst y Orth, 2009; Rehm, Rehm, Shield, Gmel y Gual, 2013); 4) este patrón de consumo intensivo resulta ser más dañino que el consumo regular de alcohol (Becker, 1998; Duka et al., 2004); y 5) el cerebro adolescente parece ser más vulnerable que el adulto a los efectos perjudiciales del alcohol (Cadaveira, 2009; Spear, 2013; White y Swartzwelder, 2005). Teniendo todo ello en cuenta, la persistencia del BD durante estos años de transición a la edad adulta reviste una particular preocupación desde el punto de vista social y sanitario y ha dado lugar a una línea de investigación sobre sus consecuencias neurocognitivas de gran desarrollo en los años recientes, que se revisa en este trabajo.

\section{Método}

Se realizó una revisión bibliográfica de los estudios sobre las consecuencias neurocognitivas del BD en jóvenes y adolescentes. Para ello se llevó a cabo una búsqueda en las siguientes bases de datos: Web of Knowledge (WOK) -que incluye, entre otras, las bases MEDLINE y Current Contents Connect-, PubMed y PsycINFO. Se revisaron todos los artículos seleccionados a partir de los siguientes términos de búsqueda: ("binge drinking" OR "heavy drinking" OR "heavy episodic drinking" OR "college drinking" OR "binge drinkers" OR "heavy drinkers" OR "college drinkers") AND ("adolescents" OR "young adults" OR "college students" OR "university students"). La búsqueda estuvo confinada entre el 1 de Enero de 2000 y el 31 de Diciembre de 2013. Bajo estos términos, se obtuvieron 6761 artículos en la WOK, 2494 artículos en PubMed y 2080 artículos en PsycINFO. A partir de este número inicial de artículos, los criterios para ser incluidos en la presente revisión fueron: 1) estudios realizados en humanos, 2) que exploren los efectos del BD a nivel cerebral por medio de técnicas neuropsicológicas, psicofisiológicas o de neuroimagen, 3) que los sujetos objeto de estudio tengan una edad comprendida entre los 12 y los 30 años y 4) que los sujetos evaluados no presenten Trastorno por Consumo de Alcohol (TCA).

\section{Resultados}

A partir de los términos iniciales de búsqueda, así como de los filtros posteriores, se obtuvieron un total de 36 artículos, de los cuales 13 eran estudios neuropsicológicos, cinco de neuroimagen estructural, seis de neuroimagen funcional y 12 de corte psicofisiológico. Se revisaron también diversos estudios incluidos en las referencias bibliográficas de los principales artículos sobre $\mathrm{BD}$, obteniéndose cuatro artículos más (dos neuropsicológicos, uno de neuroimagen funcional y uno psicofisiológico) que cumplían los criterios de inclusión. De este modo, el número de artículos que constituyeron el cuerpo de esta revisión fueron un total de 40. Las características más importantes así como los principales resultados y conclusiones de estos estudios se resumen en las tablas 1, 2, 3 y 4.

\section{Discusión}

\section{Consecuencias neuropsicológicas del consumo intensivo de alcohol}

Atención. Tres de los 15 estudios neuropsicológicos analizados en la presente revisión (ver Tabla 1), correspondientes a tres grupos de investigación diferentes, evaluaron de forma directa los procesos de tipo atencional. Sus resultados coinciden en señalar diferencias de desempeño entre los grupos control y $\mathrm{BD}$ en los distintos tipos de atención estudiados. Respecto a la atención focalizada, los jóvenes BDs muestran menor rendimiento que sus pares control en tareas de span atencional auditivo y visual (orden directo de Dígitos y Localización Espacial de la Wechsler Memory Scale-III, WMS-III) (Sanhueza, García-Moreno y Expósito, 2011).

El rendimiento de los BDs en tareas de atención sostenida también se diferencia del presentado por los jóvenes sin este patrón de consumo de alcohol. En el Paced Auditory Serial Addition Test (PASAT), el grupo BD no alcanza el nivel de rendimiento de su grupo de comparación (abstemios) (Hartley, Elsabagha y File, 2004). Estos resultados también se han observado mediante la administración de una tarea de vigilancia (Gordon Diagnostic System), si bien en este caso las diferencias se limitan a las mujeres $\mathrm{BD}$, sin que se aprecien diferencias en los varones (Townshend y Duka, 2005).

Memoria. Los estudios sobre la capacidad mnésica en los jóvenes $\mathrm{BD}$ suman un total de nueve publicaciones, provenientes de cuatro grupos de investigación. Por medio de diferentes tareas, en estos estudios se ha explorado la memoria declarativa episódica verbal y visual y la memoria prospectiva. 
Tabla 1

Estudios sobre el perfil neuropsicológico asociado al binge drinking en jóvenes y adolescentes

\begin{tabular}{|c|c|c|c|c|c|}
\hline Estudio & Grupos & Edad & Criterio BD & Objetivos & Tarea \\
\hline $\begin{array}{l}\text { Hartley et } \\
\text { al., } 2004\end{array}$ & $\begin{array}{l}\cdot 13 \mathrm{Cs}(6 \hat{0}, 7 \text { 이 } \\
\cdot 14 \mathrm{BDs}(9 \hat{0}, 5+)\end{array}$ & 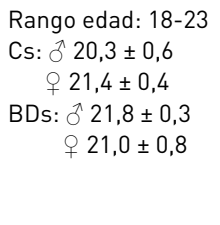 & $\begin{array}{l}>10 \text { bbs en la misma ocasión } \\
\geq 24 \text { puntos en el } A \cup Q \text {. }\end{array}$ & $\begin{array}{l}\text { Estudiar el desempeño } \\
\text { cognitivo en jóvenes BDs lasí } \\
\text { como su nivel de depresión y } \\
\text { ansiedad ). }\end{array}$ & $\begin{array}{l}\text { PASAT, Test de memoria } \\
\text { a largo plazo verbal y } \\
\text { visoespacial y } \\
\text { CANTAB (Pattern } \\
\text { Recognition Memory, Spatial } \\
\text { Recognition Memory, Spatial } \\
\text { Working Memory, SoC, IED) }\end{array}$ \\
\hline
\end{tabular}

$\begin{array}{lll}\begin{array}{l}\text { Townshend } \\ \text { y Duka, }\end{array} & \cdot 34 \mathrm{Cs}(13 \hat{0}, 21 \text { 우 } & \text { Rango edad: } 18-30 \geq 24 \text { puntos en el AUQ. } \\ 2005 & & \text { Cs: } 20,9 \pm 2,5 \\ & \text { BDs: } 20,9 \pm 2,6\end{array}$
et al., 2007

\section{BDs ligeros o}

no-BDs $(25 \hat{\jmath}, 25$ + $)$

. 50 BDs moderados

(25․, 25ㅇ)

.50 BDs "en

aumento" (25)

25이

. $50 \mathrm{BDs}$ intensivos

estables $(25 \hat{\jmath}, 25$ 우

Rango edad: $19-20$
BDs ligeros: 19,9
$\pm 0,4$
BDs moderados:
$19,9 \pm 0,3$
BDs "en aumento":
$20 \pm 0,3$
BDs intensivos
estables: $20 \pm 0,4$

5 UBEs/ocasión

$\geq 2$ veces/últimos 30 días.

- BDs ligeros: baja frecuencia de

T1 a T5.

- BDs moderados: moderada

frecuencia de T1 a T5.

- BDs "en aumento": baja

frecuencia en T1 pero creciente

de T2 a T5

- BDs intensivos: alta frecuencia

de T1 a T5.

Participantes quienes nunca

consumieron 5 UBEs en la misma

ocasión o lo hicieron 1 vez en el

último mes fueron considerados

como no-BDs o BDs ligeros.

\begin{tabular}{|c|c|c|c|}
\hline $\begin{array}{l}\text { García- } \\
\text { Moreno et } \\
\text { al., } 2008\end{array}$ & $\begin{array}{l}22 \mathrm{Cs} \\
\cdot 18 \mathrm{BDs} \text { moderados } \\
\cdot 20 \mathrm{BDs} \text { intensivos } \\
\text { Total: } 10 \hat{2}, 52+; \\
\text { distribución de los } \\
\text { grupos según sexo } \\
\text { no disponible. }\end{array}$ & $\begin{array}{l}\text { Rango edad: n.d. } \\
\text { Cs y BDs: } 18,8 \pm 1,1\end{array}$ & $\begin{array}{l}\text { BDs moderados: } \\
\leq 4 \text { UBEs en la misma ocasión. } \\
\text { - BDs intensivos: } \\
\geq 5 \text { UBEs en la misma ocasión. }\end{array}$ \\
\hline $\begin{array}{l}\text { Johnson et } \\
\text { al., } 2008\end{array}$ & 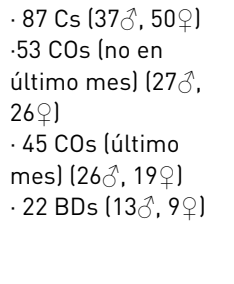 & $\begin{array}{l}\text { Rango edad: } \mathrm{n} . \mathrm{d} \text {. } \\
\text { Cs: } 16,11 \pm 0,5 \\
\text { C0s (no en último } \\
\text { mes): } 16,17 \pm 0,5 \\
\text { C0s (último mes): } \\
\text { 16,53 } \pm 0,7 \\
\text { BDs: } 16,0 \pm 0,6\end{array}$ & $\begin{array}{l}\text { COs (no en último mes): } \\
\text { consumieron alcohol, pero no en } \\
\text { el último mes. } \\
\text { - COs (último mes): } \\
<4 \text { bbs/ocasión en el último } \\
\text { mes. } \\
\text { BDs: } \\
\geq 4 \text { bbs/ocasión, } \\
\geq 1 \text { vez/mes. }\end{array}$ \\
\hline
\end{tabular}

\begin{tabular}{|c|c|c|c|}
\hline $\begin{array}{l}\text { García- } \\
\text { Moreno et } \\
\text { al., } 2009\end{array}$ & $\begin{array}{l}17 \mathrm{Cs} \text { } 9 \\
\cdot 18 \mathrm{BDs} \text { + } \\
\text { moderadas } \\
.17 \mathrm{BDs} \text { } 9\end{array}$ & $\begin{array}{l}\text { Rango edad: } \mathrm{n} . \mathrm{d} \\
\text { Cs y BDs: } 18,2 \pm 1,1\end{array}$ & $\begin{array}{l}\text { - BDs moderadas: } \\
\leq 4 \text { UBEs en la misma ocasión. } \\
\text { - BDs intensivas: } \\
\geq 5 \text { UBEs en la misma ocasión. }\end{array}$ \\
\hline
\end{tabular}

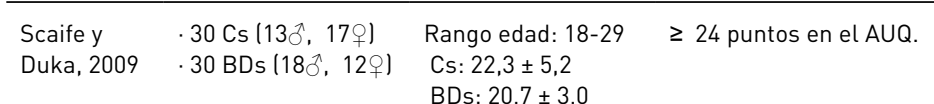

\author{
Observar la relación entre \\ patrones de consumo \\ de alcohol, desempeño \\ cognitivo, humor, \\ expectativas sobre el \\ alcohol y características de \\ personalidad.

\section{CANTAB (Matching to} \\ Sample Visual Search, \\ Spatial Working Memoryl, \\ GDS (Tarea de vigilancia)
}

\title{
Analizar la asociación
} entre la trayectoria del BD

y la toma de decisiones, así como la relación entre impulsividad y toma de

decisiones.

IGT original

Comprobar los

efectos del BD sobre

determinadas capacidades

neuropsicológicas vinculada

a la actividad neural de la

corteza prefrontal.

\section{Evaluar la toma de}

decisiones y la memoria de

trabajo en adolescentes BDs.
TAVEC, WMS-III (Dígitos),

Cubos de Corsi, Stroop,

Torre de Hanoi, Recitado de

Series (numéricas).

IGT original e IGT modificado, SOPT

\begin{abstract}
Valorar el rendimiento
neuropsicológico en jóvenes BDs.

TAVEC, WMS-III (Dígitos),

Cubos de Corsi, Stroop,

Torre de Hanoi, Recitado de Series (numéricas), BVRT.
\end{abstract}


BDs $\downarrow$ rendimiento en: atención sostenida, memoria visoespacial demorada y planificación (mayor tiempo de planificación inicial).

BDs $\hat{o} \uparrow$ tiempo de reacción en memoria de trabajo visoespacial (Spatial Recognition Memoryl que Cs $\widehat{\partial}$.

BDs $q \downarrow$ tiempo de reacción en memoria de trabajo visoespacial (Spatial Recognition Memoryl que Cs . .

BDs $\hat{\partial} \downarrow$ número de errores en etapa de cambio extradimensional en flexibilidad cognitiva (IED; CANTAB) que Cs $\widehat{\partial}$.

No diferencias en el test de memoria a largo plazo verbal ni en Pattern Recognition Memory.

BDs $\downarrow$ tiempo de elección y movimiento en tarea de búsqueda visual. BDs $\downarrow \downarrow$ rendimiento en tareas de memoria de trabajo espacial y de vigilancia que $\mathrm{Cs}$ ?.

El BD durante la juventud puede conducir a un menor rendimiento en tareas que implican atención sostenida y memoria episódica visoespacial. Igualmente, ejerce ciertos desajustes en la planificación (tiempo inicial), sin lograr afectar su precisión.

Además, el BD interfiere en los procesos de memoria de trabajo visoespacial y de flexibilidad cognitiva de forma diferenciada entre los sexos.

$\mathrm{BDs}$ intensivos estables menos elecciones ventajosas que BDs ligeros o no-BDs.

No relación entre impulsividad y nivel de rendimiento en el IGT.
Los BDs (especialmente las mujeres) presentan peor rendimiento en tareas que evalúan la memoria de trabajo visoespacial y la atención sostenida. La combinación de factores relacionados con el BD (bajo estado de ánimo y pobre funcionamiento cognitivol podría contribuir a la progresión hacia el abuso de alcohol.
El BD se relaciona con toma de decisiones desventajosas. Esta relación es independiente de la impulsividad y parece atribuirse a la edad temprana de inicio del BD, pero no a la edad de inicio de consumo de alcohol en general.
BDs $\uparrow$ número de intromisiones y perseveraciones (TAVEC) y $\downarrow$ rendimiento en el $1^{\circ}$ ensayo del recuerdo inmediato en TAVEC, así como en Dígitos, Cubos de Corsi, Recitado de Series y Stroop.

No diferencias en Torre de Hanoi.
El patrón BD provoca un deterioro neurocognitivo y neuroconductual similar en muchos aspectos al observado en bebedores crónicos.
BDs $\downarrow$ rendimiento en IGT original.

Más bajo rendimiento en IGT predecía el BD.

No diferencias en IGT modificado ni en SOPT.
Los BDs presentan hipersensibilidad al refuerzo inmediato, cuyo mecanismo neural subyacente puede actuar como factor predisponente para futuras conductas abusivas.
BDs moderadas e intensivas:

$\uparrow$ número de intromisiones y perseveraciones (en el recuerdo) y de falsos positivos (en el reconocimiento) (TAVEC).

$\downarrow$ rendimiento en Dígitos, Cubos de Corsi, Recitado de Series, Stroop y TAVEC (recuerdo inmediato [ensayo I], recuerdo inmediato total [ensayos I a V] y recuerdo libre a corto plazo [ensayo VI]]. No diferencias en Torre de Hanoi ni en BVRT.

BDs $\downarrow$ tiempo de ejecución de movimientos en la tarea de tiempo de reacción. BDs $\downarrow$ desempeño en tarea de aprendizaje visoespacial.

$\mathrm{BDs}$ ㅇ $\downarrow$ rendimiento que $\mathrm{Cs}$ 9 en flexibilidad cognitiva y memoria de trabajo espacial.
El BD ocasiona déficits neurocognitivos len atención, memoria episódica y planificación) en mujeres jóvenes que pueden equipararse a los encontrados en varones con un historial de consumo de mayor duración y frecuencia. 


\begin{tabular}{|c|c|c|c|c|c|}
\hline Estudio & Grupos & Edad & Criterio BD & Objetivos & Tarea \\
\hline $\begin{array}{l}\text { Xiao et al., } \\
2009\end{array}$ & 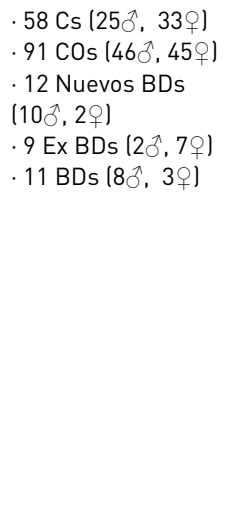 & $\begin{array}{l}\text { Rango edad: } n . d \text {. } \\
\text { Cs: } 16,2 \pm 0,6 \\
\text { COs: } 16,2 \pm 0,5 \\
\text { Nuevos BDs: } 16,4 \\
\pm 0,8 \\
\text { Ex BDs: } 16,0 \pm 0,5 \\
\text { BDs: } 16,1 \pm 0,7\end{array}$ & $\begin{array}{l}\text { COs: } \\
\text { En T1 y/o T2 consumieron alcohol } \\
\text { lalgunos participantes, en los } \\
\text { últimos } 30 \text { días), pero }<4 \text { bbs/ } \\
\text { ocasión. } \\
\text { - Nuevos BDs: } \\
\text { En T1 consumían < } 4 \text { bbs/ } \\
\text { ocasión en los últimos } 30 \text { días } \\
\text { pero en } T 2 \text { consumían } \geq 4 \text { bbs/ } \\
\text { ocasión, } \geq 1 \text { vez/mes. } \\
\text { - Ex BDs: } \\
\text { En T1, pero no en T2, consumían } \\
\geq 4 \text { bbs/ocasión, } \geq 1 \text { vez/mes. } \\
\text { BDs: } \\
\text { En T1 y T2 consumían } \geq 4 \text { bbs/ } \\
\text { ocasión, } \geq 1 \text { vez/mes. }\end{array}$ & $\begin{array}{l}\text { Evaluar la capacidad } \\
\text { predictora de la toma de } \\
\text { decisiones sobre el consumo } \\
\text { de alcohol en adolescentes. }\end{array}$ & IGT, SOPT \\
\hline $\begin{array}{l}\text { Heffernan } \\
\text { et al., } 2010\end{array}$ & $\begin{array}{l}\cdot 29 \mathrm{Cs}(5 \hat{\jmath}, 24+1) \\
\cdot 21 \mathrm{BDs}(7 \hat{\jmath}, 14+)\end{array}$ & $\begin{array}{l}\text { Rango edad: } 17-19 \\
\text { Cs: } 18,6 \pm 0,5 \\
\text { BDs: } 18,7 \pm 0,5\end{array}$ & $\begin{array}{l}\geq 8 / 6(\delta / \text { / ) bbs/ocasión } \\
\geq 2 \text { veces/semana. }\end{array}$ & $\begin{array}{l}\text { Evaluar la memoria } \\
\text { prospectiva cotidiana en } \\
\text { jóvenes BDs. }\end{array}$ & PRMQ, PRVP \\
\hline $\begin{array}{l}\text { Mullan et } \\
\text { al., } 2011\end{array}$ & $\begin{array}{l}.74 \mathrm{Cs} \\
.50 \mathrm{CMs} \\
.29 \mathrm{BDs} \\
(74 \% \text { + })\end{array}$ & $\begin{array}{l}\text { Rango edad: } n . d . \\
\text { Cs, CMs y BDs: } 20,1 \\
\pm 4,2\end{array}$ & $\begin{array}{l}\text { CMs: } \\
<5 \text { bbs/ocasión } \\
\text { BDs: } \\
\geq 5 \text { bbs/ocasión }\end{array}$ & $\begin{array}{l}\text { Determinar qué aspectos } \\
\text { de las funciones ejecutivas } \\
\text { pueden distinguir a los } \\
\text { BDs de los Cs y establecer } \\
\text { el papel de las funciones } \\
\text { ejecutivas en la predicción de } \\
\text { episodios de BD. }\end{array}$ & $\begin{array}{l}\text { Torre de Hanoi, Stroop, IGT, } \\
\text { WCST }\end{array}$ \\
\hline $\begin{array}{l}\text { Sanhueza } \\
\text { et al., } 2011\end{array}$ & 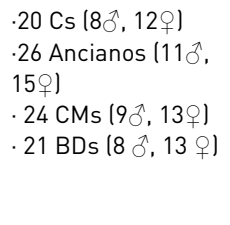 & $\begin{array}{l}\text { Rango edad: n.d. } \\
\text { Cs: } 18,8 \pm 1,7 \\
\text { Ancianos: } 69,3 \pm 4,8 \\
\text { CMs: } 19,0 \pm 1,4 \\
\text { BDs: } 19,0 \pm 1,2\end{array}$ & 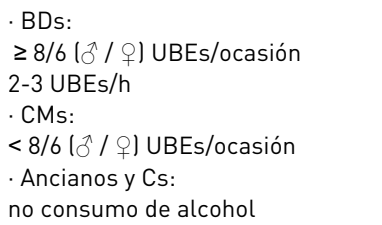 & $\begin{array}{l}\text { Investigar si el consumo } \\
\text { abusivo de alcohol en } \\
\text { jóvenes provoca alteraciones } \\
\text { cognitivas comparables a las } \\
\text { encontradas en personas de } \\
\text { edad avanzada. }\end{array}$ & $\begin{array}{l}\text { TAVEC, WMS-III (Dígitos } \\
\text { y Localización Espacial), } \\
\text { Stroop, Torre de Hanoi, BVRT }\end{array}$ \\
\hline
\end{tabular}

\begin{tabular}{|c|c|c|c|c|c|}
\hline $\begin{array}{l}\text { Parada et } \\
\text { al., 2011b }\end{array}$ & $\begin{array}{l}.60 \mathrm{Cs}(31 \hat{0}, 29+1) \\
.62 \mathrm{BDs}(30 \hat{0}, 32+)\end{array}$ & 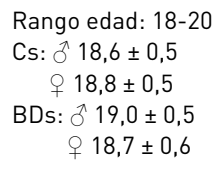 & $\begin{array}{l}\geq 6 \text { UBEs/ocasión } \\
\geq 1 \mathrm{vez} / \mathrm{mes} \\
\geq 3 \mathrm{bbs} / \mathrm{h} \\
0 \text { bien: } \\
\geq 6 \text { UBEs/ocasión } \\
\geq 1 \mathrm{vez} / \text { semana }\end{array}$ & $\begin{array}{l}\text { Examinar la relación entre } \\
\text { BD y memoria declarativa en } \\
\text { estudiantes universitarios. }\end{array}$ & $\begin{array}{l}\text { RAVLT, WMS-III (Textos I-II, } \\
\text { Escenas I-II) }\end{array}$ \\
\hline $\begin{array}{l}\text { Parada et } \\
\text { al., } 2012\end{array}$ & $\begin{array}{l}.60 \mathrm{Cs}(31 \hat{\jmath}, 29+) \\
.62 \mathrm{BDs}(30 \hat{\jmath}, 32+)\end{array}$ & 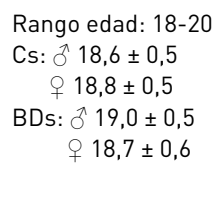 & $\begin{array}{l}\geq 6 \text { UBEs/ocasión } \\
\geq 1 \mathrm{vez} / \mathrm{mes} \\
\geq 3 \mathrm{bbs} / \mathrm{h} \\
0 \text { bien: } \\
\geq 6 \text { UBEs/ocasión } \\
\geq 1 \mathrm{vez} / \text { semana }\end{array}$ & $\begin{array}{l}\text { Analizar la relación entre } \\
\text { BD y funciones cognitivas } \\
\text { dependientes del córtex } \\
\text { prefrontal en estudiantes } \\
\text { universitarios. }\end{array}$ & $\begin{array}{l}\text { WMS-III (Dígitos y } \\
\text { Localización Espacial), SOPT, } \\
\text { Fluencia verbal (PMR), BADS } \\
\text { (Mapa del Zoo y Búsqueda de } \\
\text { Llaves), WCST-3 }\end{array}$ \\
\hline $\begin{array}{l}\text { Sneider et } \\
\text { al., } 2013\end{array}$ & $\begin{array}{l}\cdot 29 \mathrm{Cs}(15 \hat{\jmath}, 14+1) \\
\cdot 22 \mathrm{BDs}(13 \hat{\delta}, 9+1)\end{array}$ & $\begin{array}{l}\text { Rango edad: } \text { n.d. } \\
\text { Cs: } 21,5 \pm 1,7 \\
\text { BDs: } 22,1 \pm 1,3\end{array}$ & 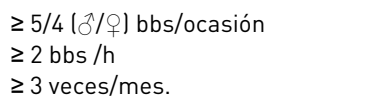 & $\begin{array}{l}\text { Evaluar el efecto del BD en } \\
\text { la memoria en los jóvenes. }\end{array}$ & $\begin{array}{l}\text { CVLT y análogo humano del } \\
\text { WMT }\end{array}$ \\
\hline $\begin{array}{l}\text { Mota et al., } \\
2013\end{array}$ & 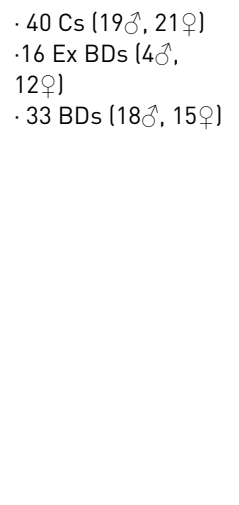 & $\begin{array}{l}\text { Inicio: } \\
\text { Rango edad: n.d. } \\
\text { Cs: } 18,5 \pm 0,5 \\
\text { Ex BDs: } 18,9 \pm 0,6 \\
\text { BDs: } 18,8 \pm 0,6 \\
\text { Seguimiento: } \\
\text { iRango edad: n.d. } \\
\text { Cs: } 20,4 \pm 0,6 \\
\text { Ex BDs: } 20,6 \pm 0,5 \\
\text { BDs: } 20,6 \pm 0,8\end{array}$ & $\begin{array}{l}\text { Ex BDs: } \\
\text { En T1, pero no en T2, consumo } \geq \\
6 \text { UBEs/ocasión } \\
\geq 1 \text { vez/mes } \\
\geq 3 \mathrm{bbs/h} \\
0 \text { bien: } \\
\geq 6 \text { UBEs/ocasión } \\
\geq 1 \text { vez/semana. } \\
\text { BDs: } \\
\text { En } 11 \text { y T2 consumo } \geq 6 \text { UBEs/ } \\
\text { ocasión } \\
\geq 1 \text { vez/mes } \\
\geq 3 \text { bbs/h } \\
0 \text { bien: } \\
\geq 6 \text { UBEs/ocasión } \\
\geq 1 \text { vez/semana. }\end{array}$ & $\begin{array}{l}\text { Investigar la relación } \\
\text { entre la trayectoria del BD } \\
\text { durante la adolescencia y } \\
\text { juventud y el funcionamiento } \\
\text { neuropsicológico. }\end{array}$ & $\begin{array}{l}\text { RAVLT, WMS-III (Textos I-II, } \\
\text { Escenas I-II, span atencional } \\
\text { en orden inverso de Dígitos } \\
\text { y Localización Espacial), } \\
\text { SOPT, BADS (Mapa del Zoo y } \\
\text { Búsqueda de Llaves) }\end{array}$ \\
\hline
\end{tabular}

Nota. AUQ: alcohol use questionnaire; BADS: behavioral assessment of dysexecutive syndrome; bbs: bebidas alcohólicas; BD: binge drinking; BDs: binge drinkers; BVRT: Benton's visual retention test; Cs: Controles; CAMPROMPT: Cambridge prospective memory test; CANTAB: Cambridge neuropsychological test automated battery; CAS: concentración de alcohol en sangre; CMs: consumidores moderados; COs: consumidores ocasionales; CVLT: California verbal learning test; GDS: Gordon diagnostic system; IAT: implicit association test; IED: intra-extradimensional shift task; IGT: lowa gambling task; ITD: Imágenes por Tensor 


\section{Principales resultados}

BDs y nuevos BDs $\downarrow$ rendimiento en IGT que $\mathrm{Cs}, \mathrm{CO}$ s y Ex BDs. Mejores puntuaciones en IGT (pero no en el SOPT) predecían menores problemas relacionados con el alcohol y menor consumo 1 año después. No diferencias en el SOPT.

\section{Conclusiones}

Déficits en toma de decisiones podrían ser determinantes del consumo intensivo de alcohol así como de otras conductas adictivas en los adolescentes.
BDs $\downarrow$ recuerdo de combinaciones localización-acción que los Cs. No diferencias en lapsus de memoria prospectiva autoinformados.
El BD durante la adolescencia y juventud provoca disfunción en la memoria prospectiva cotidiana.
$\mathrm{BDs} \downarrow$ rendimiento en IGT que $\mathrm{Cs}$.

CMs $\uparrow$ puntuación en Stroop que Cs.

BDs $\downarrow$ rendimiento en Torre de Hanoi que CMs.

No diferencias entre grupos en WCST.

Las puntuaciones en Torre de Hanoi y en Stroop, en interacción con la intención de consumo intensivo de alcohol, predicen los episodios de BD.
El BD puede afectar a la toma de decisiones en los jóvenes (los BDs toman decisiones menos ventajosas que los Cs).

La mayor capacidad de planificación y de control inhibitorio de los consumidores moderados en comparación con los BDs sugiere que el entrenamiento en estas funciones cognitivas puede ser una estrategia útil para reducir o prevenir el BD.
Perseveraciones (TAVEC):

BDs y CMs $\uparrow$ número de perseveraciones que Cs y Ancianos.

Span verbal y espacial (orden directo Dígitos y Localización Espacial): Ancianos $\downarrow$ desempeño que BDs y CMs; BDs y CMs $\downarrow$ rendimiento que $\mathrm{Cs}$. Stroop (palabra)

BDs, CMs y Ancianos $\downarrow$ desempeño que Cs.

Stroop (color y palabra/color): Ancianos $\downarrow$ desempeño que BDs y CMs; BDs y

CMs $\downarrow$ rendimiento que Cs.

Torre de Hanoi y BVRT:

No diferencias entre los grupos jóvenes.
BDs $\uparrow$ interferencia y número de perseveraciones (RAVLT) y $\downarrow$ recuerdo inmediato y demorado (Textos I-II).

No diferencias en Escenas I-II ni en puntuaciones de recuerdo en RAVLT.
Ciertas similitudes entre los resultados obtenidos por los jóvenes bebedores y los mayores están en línea con la hipótesis del envejecimiento prematuro. El menor rendimiento de los jóvenes bebedores se daba en tareas relacionadas con las funciones ejecutivas y, por lo tanto, con el córtex prefrontal.
$\mathrm{BDs} \uparrow$ número de perseveraciones (SOPT).

$\mathrm{BD} \hat{\jmath} \downarrow$ rendimiento en span verbal en orden inverso (Dígitos) que $\mathrm{BD}$ \& y $\mathrm{Cs}$

No diferencias en Localización Espacial (WMS-III), fluencia verbal (PMR), BADS (Mapa del Zoo y Búsqueda de Llaves) yWCST-3.
El patrón BD está asociado con desempeño inferior en funciones ejecutivas dependientes del córtex prefrontal dorsolateral. Estas dificultades pueden reflejar retraso neuromadurativo o disfunción en el lóbulo frontal.
BDs $\uparrow$ número de perseveraciones (SOPT) y $\downarrow$ recuerdo inmediato y demorado (Textos I-II).

No diferencias en puntuaciones de recuerdo en RAVLT, span atencional en orden inverso de Dígitos y Localización Espacial, Escenas I-II, Mapa del Zoo y Búsqueda de Llaves (BADS).
La persistencia, pero no el abandono, en el BD se asocia a dificultades en memoria verbal y supervisión de la respuesta, procesos dependientes del córtex prefrontal dorsolateral y del córtex témporo-mesial. 
Tabla 2

Estudios neuroestructurales en la población de jóvenes y adolescentes binge drinkers.

\begin{tabular}{|c|c|c|c|c|c|}
\hline Estudio & Grupos & Edad & Criterio BD & Objetivos & Técnica/Tarea \\
\hline $\begin{array}{l}\text { Jacobus et } \\
\text { al., } 2009\end{array}$ & 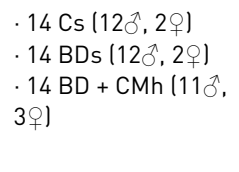 & $\begin{array}{l}\text { Rango edad: } 16-19 \\
\text { Cs: } 17,3 \pm 0,8 \\
\text { BDs: } 18,1 \pm 0,7 \\
\text { BD + CMh: } 18,2 \\
\pm 0,7\end{array}$ & $\begin{array}{l}\geq 5 / 4\left(\delta^{\lambda} / \text { + }\right) \text { bbs en la misma } \\
\text { ocasión } \\
\geq 1 \text { vez últimos } 3 \text { meses }\end{array}$ & $\begin{array}{l}\text { Estudiar la integridad de las } \\
\text { fibras de sustancia blanca } \\
\text { en adolescentes BDs con } \\
\text { y sin consumo habitual de } \\
\text { marihuana. }\end{array}$ & $\cdot$ ITD \\
\hline $\begin{array}{l}\text { McQueeny } \\
\text { et al., } 2009\end{array}$ & $\begin{array}{l}\cdot 14 \mathrm{Cs}(12 \hat{0}, 29) \\
\cdot 14 \mathrm{BDs}(12 \hat{0}, 29)\end{array}$ & $\begin{array}{l}\text { Rango edad: } 16-19 \\
\text { Cs: } 17,9 \pm 0,9 \\
\text { BDs: } 18,1 \pm 0,7\end{array}$ & $\begin{array}{l}\geq 5 / 4(\delta / \phi) \text { bbs en la misma } \\
\text { ocasión } \\
\geq 1 \text { vez últimos } 3 \text { meses }\end{array}$ & $\begin{array}{l}\text { Examinar la integridad de las } \\
\text { fibras de sustancia blanca en } \\
\text { adolescentes BDs. }\end{array}$ & $\cdot$ ITD \\
\hline $\begin{array}{l}\text { Squeglia, } \\
\text { Sorg et al., } \\
2012\end{array}$ & $\begin{array}{l}\cdot 30 \mathrm{Cs}(15 \hat{0}, 15 q) \\
\cdot 29 \mathrm{BDs}(15 \hat{0}, 14+)\end{array}$ & $\begin{array}{c}\text { Rango edad: } 15-19 \\
\text { Cs: of } 17,9 \pm 1,1 \\
\text { o } 18,0 \pm 1,1 \\
\text { BDs: } 018,6 \pm 0,6 \\
\text { of } 17,8 \pm 12\end{array}$ & $\begin{array}{l}\geq 5 / 4(\delta / \text { l }) \text { bbs en la misma } \\
\text { ocasión } \\
\geq 1 \text { vez últimos } 3 \text { meses }\end{array}$ & $\begin{array}{l}\text { Examinar el volumen de } \\
\text { sustancia gris del CF en los } \\
\text { adolescentes BDs de ambos } \\
\text { sexos. }\end{array}$ & $\begin{array}{l}\text { - IRM } \\
\text {-Puebas neuropsicológicas } \\
\text { (D-KEFS, } \\
\text { WAIS-III, } \\
\text { Figura Compleja de Reyl }\end{array}$ \\
\hline $\begin{array}{l}\text { Lisdahl et } \\
\text { al., } 2013\end{array}$ & $\begin{array}{l}.60 \mathrm{Cs}(35 \hat{\jmath}, 25 \text { 이 } \\
.46 \mathrm{BDs}(31 \hat{\jmath}, 15 \text { 이 }\end{array}$ & $\begin{array}{l}\text { Rango edad: } 16-19 \\
\text { Cs: } 17,7 \pm 1,0 \\
\text { BDs: } 18,0 \pm 0,8\end{array}$ & $\begin{array}{l}\geq 5 / 4(\delta / O) \text { bbs en la misma } \\
\text { ocasión } \\
\geq 1 \text { vez últimos } 3 \text { meses }\end{array}$ & $\begin{array}{l}\text { Evaluar los efectos del BD en } \\
\text { el volumen del cerebelo en } \\
\text { adolescentes. }\end{array}$ & $\cdot$ IRM \\
\hline $\begin{array}{l}\text { Howell et } \\
\text { al, } 2013\end{array}$ & $\begin{array}{l}\cdot 19 \mathrm{Cs}(7 \delta, 12+) \\
\cdot 19 \mathrm{BDs}(7 \delta, 12+)\end{array}$ & $\begin{array}{l}\text { Rango edad: n.d. } \\
\text { Cs: } 24,6 \pm 4,4 \\
\text { BDs: } 22,9 \pm 3,4\end{array}$ & $\begin{array}{l}\geq 5 / 4 \text { UBEs en la misma ocasión } \\
\geq 2 \mathrm{bbs} / \mathrm{h} \\
\geq 1 \mathrm{vez} / \text { semana en los últimos } 3 \\
\text { meses }\end{array}$ & $\begin{array}{l}\text { Evaluar en estudiantes } \\
\text { universitarios BDs el } \\
\text { volumen de sustancia gris en } \\
\text { las regiones subcorticales } \\
\text { afectadas por el abuso de } \\
\text { alcohol en adolescentes. }\end{array}$ & $\cdot$ IRM \\
\hline
\end{tabular}

Nota. AF: anisotropía fraccional; AUDIT: alcohol use disorders identification test; bbs: bebidas alcohólicas; BD: binge drinking; BDs: binge drinkers; CF: córtex frontal; Cs: controles; CMh: consumidores de marihuana; D-KEFS: Delis-Kaplan executive function system; IRM: imagen por resonancia magnética; ITD: imagen por tensor de difusión; n.d.: no disponible; UBEs: unidades de bebida estándar; WAIS-III: Wechsler adult intelligence scale, 3rd edition. 


\section{Principales Resultados}

BDs $\downarrow$ AF que $C$ s en 8 clústeres localizados en la corona radiada superior, fascículo inferior fronto-occipital y fascículo longitudinal inferior y superior. $\mathrm{BDs}+\mathrm{CMh} \downarrow \mathrm{AF}$ que $\mathrm{Cs}$ en 3 de los 8 clústeres $(2$ en la corona radiada y 1 en el fascículo longitudinal inferior).

$\mathrm{BDs}+\mathrm{CMh} \uparrow \mathrm{AF}$ que BDs en 4 de los 8 clústeres (fascículo longitudinal superior, fascículo fronto-occipital inferior, corona radiada superior y pedúnculo cerebeloso medio).

$\mathrm{BDs} \downarrow \mathrm{AF}$ en fascículo longitudinal superior e inferior, corona radiada, cápsula interna y externa, cuerpo calloso, cerebelo y fibras de proyección límbica. Más ocasiones de abstinencia y resaca correlacionan con $\downarrow$ anisotropía fraccional en el cuerpo calloso.

Mujeres BDs $\uparrow$ densidad del CF izdo. (polo frontal y orbital, giro orbitomedial y región rostral anterior del giro cinguladol que correlacionó con peor ejecución en pruebas de planificación visoespacial, inhibición y atención. Hombres BDs $\downarrow$ densidad del CF izdo. (polo orbital, giro orbitomedial y región rostral anterior del giro cingulado); no obstante, a mayor densidad cortical peor ejecución en pruebas de atención.

\section{Conclusiones}

El BD puede conducir a alteraciones en fibras de sustancia blanca importantes para el desarrollo cognitivo.
BDs $\downarrow$ densidad del cerebelo tanto en la sustancia gris como en la sustancia blanca de ambos hemisferios.

No se observaron diferencias de sexo y patrones BD más intensos correlacionan con menor volumen cerebelar.

$\mathrm{BDs} \uparrow$ volumen de sustancia gris en el núcleo estriado ventral, aunque no diferencias en hipocampo y amígdala, en comparación con controles.

Correlación negativa del volumen de la sustancia gris del núcleo estriado ventral, hipocampo y amígdala con la puntuación del test AUDIT.
Adolescentes BDs presentan reducciones generalizadas de AF en las principales vías de sustancia blanca. Aunque preliminares, estos resultados podrían indicar que la exposición a un patrón BD durante la juventud puede comprometer la integridad de las fibras de la sustancia blanca.

El BD durante la adolescencia se asocia con diferencias en función del sexo en la densidad de sustancia gris del CF que correlacionan con peor desempeño de las funciones ejecutivas, principalmente en las mujeres. Estos resultados sugieren un posible retraso neuromadurativo en las mujeres BDs, las cuales podrían ser más susceptibles a los efectos adversos del alcohol.

El BD en los últimos tres meses predice menor volumen cerebelar en los adolescentes.

Posible retraso neuromadurativo en los jóvenes $\mathrm{BDs}$, que podría relacionarse con efectos compensatorios derivados del BD o con un factor de riesgo endofenotípico. 
Tabla 3

Estudios con neuroimagen funcional en la población de jóvenes y adolescentes binge drinkers.

\begin{tabular}{|c|c|c|c|c|c|}
\hline Estudio & Grupos & Edad & Criterio BD & Objetivos & Tarea \\
\hline $\begin{array}{l}\text { Schweinsburg } \\
\text { et al., } 2010\end{array}$ & $\begin{array}{l}\cdot 12 \mathrm{Cs}\left(10 \delta^{\lambda}, 2+\right) \\
\cdot 12 \mathrm{BDs}\left(80^{\lambda}, 4+9\right)\end{array}$ & $\begin{array}{l}\text { Rango edad: } 16-18 \\
\text { Cs: } 18,2 \pm 0,8 \\
\text { BDs: } 17,8 \pm 0,9\end{array}$ & $\begin{array}{l}\geq 5 / 4(\hat{\jmath} /(\text { ) }) \text { bbs en la misma } \\
\text { ocasión } \\
\geq 1 \text { vez últimos } 3 \text { meses }\end{array}$ & $\begin{array}{l}\text { Estudiar la respuesta neural } \\
\text { durante la codificación verbal } \\
\text { en adolescentes BDs. }\end{array}$ & $\begin{array}{l}\text { Tarea de aprendizaje verbal } \\
\text { de pares asociados }\end{array}$ \\
\hline
\end{tabular}

\begin{tabular}{|c|c|c|c|}
\hline $\begin{array}{l}\text { Schweinsburg } \\
\text { et al., } 2011\end{array}$ & $\begin{array}{l}\cdot 22 \mathrm{Cs}(16 \hat{\jmath}, 6+) \\
\cdot 16 \mathrm{BDs}(13 \hat{\jmath}, 3+) \\
\cdot 8 \mathrm{CMh}(4 \hat{\jmath}, 4 q) \\
\cdot 28 \mathrm{BDs}+\mathrm{CMh} \\
(23 \hat{\jmath}, 5+)\end{array}$ & $\begin{array}{l}\text { Rango edad: } 16-18 \\
\text { Cs: } 17,6 \pm 0,8 \\
\text { BDs: } 18,1 \pm 0,7 \\
\text { CMh: } 18,1 \pm 0,9 \\
\text { BDs + CMh: } 18,0 \\
\pm 1,0\end{array}$ & $\begin{array}{l}\geq 5 / 4(\hat{\jmath} / q) \text { bbs en la misma } \\
\text { ocasión } \\
\geq 1 \text { vez últimos } 3 \text { meses }\end{array}$ \\
\hline $\begin{array}{l}\text { Squeglia et al., } \\
2011\end{array}$ & $\begin{array}{l}\cdot 55 \mathrm{Cs}(31 \delta, 24+) \\
\cdot 40 \mathrm{BDs}(27 \delta, 13+)\end{array}$ & 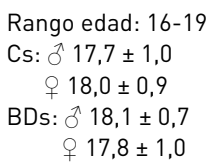 & $\begin{array}{l}\geq 5 / 4(\hat{\jmath} /(+)) \text { bbs en la misma } \\
\text { ocasión } \\
\geq 1 \text { vez últimos } 3 \text { meses }\end{array}$ \\
\hline
\end{tabular}

Examinar la respuesta cerebral de los adolescentes BDs y de los CMh durante el aprendizaje verbal.

- Tarea de aprendizaje verbal de pares asociados

\begin{tabular}{|c|c|c|c|c|c|}
\hline $\begin{array}{l}\text { Squeglia et al., } \\
2012\end{array}$ & $\begin{array}{l}\text { Estudio 1: } \\
\cdot 20 \mathrm{Cs}(14 \hat{\jmath}, 6+) \\
\cdot 20 \mathrm{BDs}(14 \hat{0}, 6+) \\
\text { Estudio 2: } \\
\cdot 20 \mathrm{Cs}(14 \hat{\jmath}, 6+) \\
\cdot 20 \mathrm{BDs}(14 \hat{\jmath}, 6+)\end{array}$ & $\begin{array}{l}\text { Estudio 1: } \\
\text { Rango edad: } 15-19 \\
\text { Cs: n.d. } \\
\text { BDs: } 17,6 \pm 1,3 \\
\text { Estudio } 2 \text { : } \\
\text { Inicio: } \\
\text { Rango edad: } 12-16 \\
\text { Cs: } 14,7 \pm 1,1 \\
\text { BDs: } 15,1 \pm 1,3 \\
\text { Seguimiento: } \\
\text { Rango edad: } 15-18 \\
\text { Cs: } 17,7 \pm 1,4 \\
\text { BDs: } 18,5 \pm 1,9\end{array}$ & $\begin{array}{l}\text { Últimos } 3 \text { meses: } \\
\cdot 1-2 \text { bbs diariamente } \\
\cdot 1-2 \text { bbs más de } 8 \text { veces } / \text { mes, } n^{0} \\
\text { bbs por episodio }=3-4 \\
\cdot 1-2 \text { bbs } 4-8 \text { veces } / \text { mes, } n^{0} \text { bbs } \\
\text { por episodio }>4 \\
\cdot 3-4 \text { bbs } 4-8 \text { veces } / \text { mes, } n^{0} \text { bbs } \\
\text { por episodio }=3-4 \\
\cdot 3-4 \text { bbs } 1-3 \text { veces } / \text { mes, } n^{0} \text { bbs } \\
\text { por episodio }>4 \\
\cdot 4 \text { bbs, menos de } 1 \text { vez } / \text { mes, } n^{0} \\
\text { bbs por episodio }>4 \\
\cdot>4 \text { bbs, menos de } 1 \text { vez } / \text { mes, } n^{0} \\
\text { bbs por episodio }>4\end{array}$ & $\begin{array}{l}\text { Determinar si las anomalías } \\
\text { cerebrales observadas en } \\
\text { los BDs son consecuencia } \\
\text { del BD, un factor de riesgo } \\
\text { preexistente o ambas. }\end{array}$ & $\begin{array}{l}\text { Tarea de memoria de } \\
\text { trabajo espacial }\end{array}$ \\
\hline $\begin{array}{l}\text { Xiao et al., } \\
2013\end{array}$ & $\begin{array}{l}\cdot 14 \mathrm{Cs}(5 \hat{\jmath}, 90) \\
\cdot 14 \mathrm{BDs}(8 \hat{0}, 6+)\end{array}$ & $\begin{array}{l}\text { Rango edad: } 16-18 \\
\text { Cs: } 17,1 \pm 0,7 \\
\text { BDs: } 17,3 \pm 0,5\end{array}$ & $\begin{array}{l}\geq 5 \text { bbs en la misma ocasión } \\
\geq 1 \text { vez último mes }\end{array}$ & $\begin{array}{l}\text { Estudiar los correlatos } \\
\text { neurales de la toma de } \\
\text { decisiones afectiva en } \\
\text { adolescentes BDs. }\end{array}$ & $\cdot$ IGT \\
\hline $\begin{array}{l}\text { Campanella et } \\
\text { al., } 2013\end{array}$ & $\begin{array}{l}\cdot 16 \mathrm{Cs}(7 \delta, 9+) \\
\cdot 16 \operatorname{BDs}(7 \delta, 9 q)\end{array}$ & $\begin{array}{l}\text { Rango edad: n.d. } \\
\text { Cs: } 20,9 \pm 1,8 \\
\text { BDs: } 21,6 \pm 2,6\end{array}$ & $\begin{array}{l}\geq 6 \text { UBEs en la misma ocasión } \\
2-3 \text { veces/semana } \\
\geq 2 \text { bbs/hora }\end{array}$ & $\begin{array}{l}\text { Comparar la actividad } \\
\text { cerebral de los BDs con la de } \\
\text { sus pares control durante la } \\
\text { realización de una tarea de } \\
\text { memoria de trabajo. }\end{array}$ & $\begin{array}{l}\text { Tarea de memoria de } \\
\text { trabajo n-back }\end{array}$ \\
\hline $\begin{array}{l}\text { Wetherill et } \\
\text { al., } 2013\end{array}$ & $\begin{array}{l}\cdot 20 \mathrm{Cs}(11 \delta, 99) \\
\cdot 20 \mathrm{BDs}(11 \delta, 9+1)\end{array}$ & $\begin{array}{l}\text { Inicio: } \\
\text { Rango edad: n.d. } \\
\text { Cs: } 14,1 \pm 1,2 \\
\text { BDs: } 14,7 \pm 1,1 \\
\text { Seguimiento: } \\
\text { Rango edad: } \text { n.d. } \\
\text { Cs: } 17,6 \pm 1,2 \\
\text { BDs: } 18,5 \pm 1,8\end{array}$ & $\begin{array}{l}\text { Últimos } 3 \text { meses: } \\
\cdot 1-2 \text { bb diariamente } \\
\cdot 1-2 \text { bb más de } 8 \text { veces } / \text { mes, } n^{0} \\
\text { bbs por episodio }=3-4 \\
\cdot 1-2 \text { bbs } 4-8 \text { veces } / \text { mes, } n^{0} \text { bbs } \\
\text { por episodio }>4 \\
\cdot 3-4 \text { bbs } 4-8 \text { veces } / \text { mes, } n^{0} \text { bbs } \\
\text { por episodio }=3-4 \\
\cdot 3-4 \text { bbs } 1-3 \text { veces } / \text { mes, } n^{\circ} \\
\text { bebidas por episodio }>4 \\
\cdot 4 \text { bbs, menos de } 1 \text { vez/mes, } n^{0} \\
\text { bbs por episodio }>4 \\
\cdot>4 \text { bbs, menos de } 1 \text { vez/mes, } n^{0} \\
\text { bbs por episodio }>4\end{array}$ & $\begin{array}{l}\text { Determinar si las anomalías } \\
\text { en el funcionamiento } \\
\text { cerebral durante la } \\
\text { inhibición de respuesta } \\
\text { predicen el paso hacia, o son } \\
\text { consecuencia de, un patrón } \\
\text { de consumo BD. }\end{array}$ & - Tarea Go/NoGo \\
\hline
\end{tabular}

Caracterizar las posibles influencias del género sobre la actividad cerebral relacionada con la memoria de trabajo en adolescentes BDs.

Nota. bbs: bebidas alcohólicas; BD: binge drinking; BDs: binge drinkers; Cs: controles; CMh: consumidores de marihuana; COF: córtex orbitofrontal; $\mathrm{CPI}$ : cortex parietal inferior; CPFDM: córtex prefrontal dorsomedial; GFI: giro frontal inferior; GFM: giro frontal medio; GFS: giro frontal superior; GTM: giro temporal medio; GTS: giro temporal superior; IGT: lowa gambling task; n.d.: no disponible; UBEs: unidades de bebida estándar. 


\section{Principales Resultados}

En la codificación de ítems nuevos

BDs $\uparrow$ actividad frontal superior dcha. y parietal posterior bilateral y $\downarrow$ actividad occipital.

No activación hipocámpica (si presente en Cs).

\section{Conclusiones}

Los BDs emplean más recursos neurales fronto-parietales relacionados con

la memoria de trabajo durante la codificación verbal.
En la codificación de ítems nuevos: BDs y BDs + CMh: $\uparrow$ actividad en giro GFS dcho., giro postcentral y CPI.

$\downarrow$ actividad en giro fusiforme izdo., giro precentral izdo., GFM y GFI dcho. GFM izdo. y cingulado, CPI izdo. y precúneo. Todo ello en comparación con los no $\mathrm{BD}$ (Cs y $\mathrm{CMh}$ ).
Los BDs presentan una reorganización o un mayor reclutamiento de las redes neurales implicadas en la memoria de trabajo durante la codificación verbal.
Interacción BD x Sexo en regiones frontales (GFS y GFI dcho., GFM izdo. cingulado anterior), temporales (GTS izdo., GTM dcho.) y cerebelares: $\delta$ BDs presentan $\uparrow$ actividad que $\widehat{\partial} \mathrm{C}$, lo cual correlaciona con mejor ejecución en pruebas neuropsicológicas.

으 $\mathrm{BD}$ presentan $\downarrow$ actividad que $q \mathrm{Cs}$, lo cual correlaciona con peor ejecución en pruebas neuropsicológicas.
Las mujeres pueden ser más vulnerables a los efectos neurotóxicos del BD durante la adolescencia.

\section{Estudio 1:}

$\uparrow$ actividad fronto-parietal (GFM izdo., Área Motora Suplementaria, GFM dcho., GFS dcho., CPI/Giro Supramarginal) y $\downarrow$ actividad en áreas visuales (Giro Occipital medial izdo.) en BDs en comparación con Cs

Estudio 2:

$\downarrow$ actividad fronto-parietal (GFM izdo. y CPI dcho.) relacionada con la memoria de trabajo en adolescentes no consumidores que tres años más tarde presentan un patrón BD.
El BD durante la adolescencia puede conducir a sutiles alteraciones en el funcionamiento neural: reorganización compensatoria (efecto post-consumo) Anomalías neurales previas al inicio del consumo podrían constituir un factor de riesgo para el futuro uso de sustancias (efecto pre-consumo)
BDs:

Peor rendimiento en el IGT.

$\uparrow$ actividad amígdala izda. e ínsula bilateral durante la toma de decisiones. Correlación entre el grado de problemas asociados al alcohol y la actividad en COF (negativa) y en ínsula dcha. (positiva).

BDs: $\uparrow$ actividad área motora presuplementaria durante la tarea de memoria de trabajo que en la tarea control, frente a los Cs, en los que no hay diferencia.

Correlación entre la actividad durante la condición de memoria de trabajo en CPFDM y número de bebidas por ocasión, y entre actividad en cerebelo, tálamo dcho. e ínsula dcha. y número de veces que consumen semanalmente.

En la 1 a evaluación, los futuros BDs muestran $\downarrow$ actividad que los $C$ s en parte del circuito inhibitorio (GFM bilateral, CPI dcho., putamen izdo., amígdala cerebelar izda.). Con el paso al BD, estos sujetos presentan

incremento de actividad en este circuito mientras que los $\mathrm{Cs}$ muestran decremento.

El cambio en la actividad en GFM dcho. correlacionó con el número de consumiciones a lo largo de la vida en la $2^{2}$ evaluación.

La activación en la $1^{\text {a }}$ evaluación no predijo el consumo al seguimiento.
La alteración en la actividad de los sistemas neurales relacionados con la toma de decisiones en los BDs puede constituir un marcador neurocognitivo de riesgo para el abuso de sustancias.

$\mathrm{BD}$ conduce a cambios cerebrales compensatorios que permiten un rendimiento conductual normal. Estos cambios podrían constituir marcadores de vulnerabilidad al abuso de sustancias.

Existen anomalías neurales previas al comienzo del consumo de alcohol que pueden incrementar la probabilidad de iniciarse en el BD. El BD, a su vez, puede conducir a alteraciones adicionales en el funcionamiento cerebral. Los BDs precisan más actividad neural para inhibir correctamente la respuesta. 
Tabla 4

Estudios psicofisiológicos en la población de jóvenes y adolescentes binge drinkers

\begin{tabular}{|c|c|c|c|c|c|}
\hline Estudio & Grupos & Edad & Criterio BD & Objetivos & Tarea \\
\hline $\begin{array}{l}\text { Ehlers et } \\
\text { al., } 2007\end{array}$ & $\begin{array}{l}\cdot 36 \mathrm{Cs}(15 \hat{\jmath}, 21) \\
\cdot 30 \mathrm{BDs}(15 \hat{\jmath}, 15+) \\
\cdot 59 \mathrm{BDs} \text { con } \\
\text { dependencia de } \\
\text { otras drogas }(22 \hat{\jmath}, \\
37 \text {. }\end{array}$ & $\begin{array}{l}\text { Rango edad: n.d. } \\
\text { Cs: } 19,6 \pm 2,1 \\
\text { BDs: } 19,7 \pm 2,0 \\
\text { BD + dependencia } \\
\text { otras drogas: } 20,3 \\
\pm 2,2\end{array}$ & $\begin{array}{l}\text { > } 5 \text { bbs en la misma ocasión } \\
\text { regularmente durante la } \\
\text { adolescencia }\end{array}$ & $\begin{array}{l}\text { Examinar la relación entre } \\
\text { BD durante la adolescencia } \\
\text { (con y sin dependencia } \\
\text { de otras drogas) y el } \\
\text { componente P3. }\end{array}$ & $\begin{array}{l}\text { - Tarea de discriminación } \\
\text { de expresiones faciales } \\
\text { emocionales }\end{array}$ \\
\hline
\end{tabular}

\begin{tabular}{|c|c|c|c|}
\hline $\begin{array}{l}\text { Maurage et } \\
\text { al., } 2009\end{array}$ & $\begin{array}{l}\cdot 18 \mathrm{Cs}(7 \delta, 11 \text { ) } \\
\cdot 18 \mathrm{BDs}(7 \hat{\delta}, 11+1)\end{array}$ & $\begin{array}{l}\text { Rango edad: } n . d \\
\text { Cs: } 18,2 \pm 0,3 \\
\text { BDs: } 18,2 \pm 0,4\end{array}$ & $\begin{array}{l}\text { Expectativa de consumo durante } \\
\text { los } 9 \text { meses siguientes a la } 1^{a} \\
\text { evaluación }>\text { de } 20 \text { UBEs/semana }\end{array}$ \\
\hline $\begin{array}{l}\text { Crego et } \\
\text { al., } 2009\end{array}$ & $\begin{array}{l}53 \mathrm{Cs}(27 \delta, 26+) \\
.42 \mathrm{BDs}(21 \delta, 21)\end{array}$ & $\begin{array}{l}\text { Rango edad: } 18-20 \\
\text { Cs: } 18,7 \pm 0,5 \\
\text { BDs: } 18,9 \pm 0,5\end{array}$ & $\begin{array}{l}\geq 6 \text { UBEs/ocasión } \\
\geq 1 \mathrm{vez} / \mathrm{mes} \\
\geq 3 \mathrm{bbs} / \mathrm{h} \\
0 \text { bien: } \\
\geq 6 \text { UBEs/ocasión } \\
\geq 1 \mathrm{vez} / \text { semana }\end{array}$ \\
\hline $\begin{array}{l}\text { Crego et } \\
\text { al., } 2010\end{array}$ & $\begin{array}{l}53 \mathrm{Cs}(27 \delta, 26 ㅇ) \\
\cdot 42 \mathrm{BDs}(21 \delta, 21)\end{array}$ & $\begin{array}{l}\text { Rango edad: } 18-20 \\
\text { Cs: } 18,7 \pm 0,5 \\
\text { BDs: } 18,9 \pm 0,5\end{array}$ & $\begin{array}{l}\geq 6 \text { UBEs/ocasión } \\
\geq 1 \mathrm{vez} / \mathrm{mes} \\
\geq 3 \mathrm{bbs} / \mathrm{h} \\
0 \text { bien: } \\
\geq 6 \text { UBEs/ocasión } \\
\geq 1 \mathrm{vez} / \text { semana }\end{array}$ \\
\hline $\begin{array}{l}\text { Courtney } \\
\text { y Polich, } \\
2010\end{array}$ & $\begin{array}{l}\cdot 32 \mathrm{Cs}\left(160^{\lambda}, 16+\right) \\
\cdot 32 \mathrm{BDs} \text { moderados } \\
(16 \hat{0}, 16+9) \\
\cdot 32 \mathrm{BDs} \text { intensivos } \\
(16 \hat{0}, 16+)\end{array}$ & $\begin{array}{l}\text { Rango edad: n.d. } \\
\text { Cs: } \\
\text { o } 21,8 \pm 0,8 \\
\text { o } 21,4 \pm 1,3 \\
\text { BDs moderados: } \\
\text { i } 20,5 \pm 1,0 \\
\text { o } 20,4 \pm 1,1 \\
\text { BDs intensivos: } \\
\text { i } 20,8 \pm 2,0 \\
\text { o } 19,9 \pm 1,1\end{array}$ & $\begin{array}{l}\text { BDs moderados: } \\
5 / 4-7 / 6(+/ §) \text { bbs en un intervalo } \\
\text { de } 2 \mathrm{~h} \\
>1 \text { vez últimos } 6 \text { meses } \\
\text { BDs intensivos: } \\
>10 \text { bbs en un intervalo de } 2 \mathrm{~h} \\
>1 \text { vez últimos } 6 \text { meses }\end{array}$ \\
\hline
\end{tabular}

Explorar los efectos del BD a . Tarea de juicio de valencia medio plazo (9 meses) sobre emocional auditiva los componentes de los PEs en jóvenes.

Explorar los rocesos de atención y memoria de trabajo en jóvenes BDs.

- Tarea visual de ejecución continua ldetección de estímulos consecutivos idénticos)

Evaluar los efectos del BD sobre la memoria de trabajo y sus fuentes neurales en jóvenes.

- Tarea visual de ejecución continua ldetección de estímulos consecutivos idénticos)

Examinar la actividad - EEG en reposo con ojos éctrica cerebral en reposo abiertos en jóvenes con diferente intensidad de consumo de alcohol.

\begin{tabular}{|c|c|c|c|c|}
\hline $\begin{array}{l}\text { Petit et al., } \\
2012\end{array}$ & $\begin{array}{l}\cdot 18 \mathrm{Cs}(8 \hat{\lambda}, 10+) \\
\cdot 18 \mathrm{BDs}(12 \hat{0}, 6+1)\end{array}$ & $\begin{array}{l}\text { Rango edad: } 19-26 \\
\text { Cs: } 21,9 \pm 3,1 \\
\text { BDs: } 21,3 \pm 1,7\end{array}$ & $\begin{array}{l}\geq 6 \text { UBEs/ocasión } \\
\geq 1 \text { vez/semana (máximo } 4 \text { veces/ } \\
\text { semana) } \\
\geq 3 \mathrm{bbs} / \mathrm{h}\end{array}$ & $\begin{array}{l}\text { Investigar si los BDs } \\
\text { exhiben anomalías en el } \\
\text { procesamiento de señales } \\
\text { relacionadas con el alcohol. }\end{array}$ \\
\hline
\end{tabular}

\begin{tabular}{|c|c|c|c|c|c|}
\hline $\begin{array}{l}\text { Maurage et } \\
\text { al., } 2012\end{array}$ & 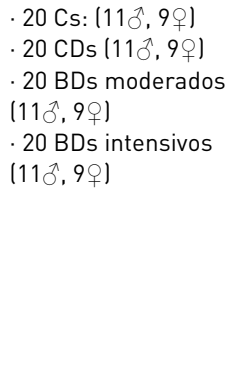 & $\begin{array}{l}\text { Rango edad: } 18-24 \\
\text { Cs: } 21,6 \pm 2,4 \\
\text { CDs: } 22,1 \pm 2,2 \\
\text { BDs moderados: } \\
21,0 \pm 2,2 \\
\text { BDs intensivos: } 21,2 \\
\pm 2,0\end{array}$ & $\begin{array}{l}\text { CDs: } \\
\text { 3-5 UBEs/ocasión, 5-7 veces/ } \\
\text { semana, < } 2 \text { UBEs/h, 15-29 UBEs/ } \\
\text { semana } \\
\text { - BDs moderados: } \\
\text { 5-12 UBEs/ocasión, 2-3 veces/ } \\
\text { semana, > } 3 \text { UBEs/h, 15-29 UBEs } \\
\text { semana } \\
\text { · BDs intensivos: } \\
>10 \text { UBEs/ocasión, 3-4 veces/ } \\
\text { semana, > } 3 \text { UBEs/h, > } 30 \text { UBEs } \\
\text { semana }\end{array}$ & $\begin{array}{l}\text { Explorar si el BD afecta } \\
\text { a las distintas etapas del } \\
\text { procesamiento visual } \\
\text { y discriminar entre la } \\
\text { influencia del consumo } \\
\text { regular de alcohol y del } \\
\text { BD sobre estos procesos } \\
\text { cognitivos. }\end{array}$ & $\begin{array}{l}\text { Tarea oddball visual cc } \\
\text { detección de caras }\end{array}$ \\
\hline $\begin{array}{l}\text { Crego et } \\
\text { al., } 2012\end{array}$ & 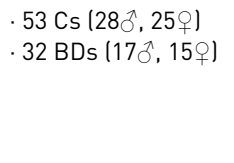 & $\begin{array}{l}\text { Rango edad: } 18-20 \\
\text { Cs: } 18,5 \pm 0,5 \\
\text { BDs: } 18,8 \pm 0,6\end{array}$ & $\begin{array}{l}\geq 6 \text { UBEs/ocasión } \\
\geq 1 \mathrm{vez} / \mathrm{mes} \\
\geq 3 \mathrm{bbs} / \mathrm{h} \\
0 \text { bien: } \\
\geq 6 \text { UBEs/ocasión } \geq 1 \mathrm{vez} / \text { semana }\end{array}$ & $\begin{array}{l}\text { Determinar si el BD afecta } \\
\text { al funcionamiento cerebral } \\
\text { relacionado con los procesos } \\
\text { de atención visual. }\end{array}$ & - Tarea oddball visual \\
\hline
\end{tabular}




Principales Resultados Conclusiones

P350:

$\downarrow$ latencia en BDs que en Cs ante expresiones faciales de felicidad, neutras y de tristeza.

$\downarrow$ latencia en BDs codependientes que en Cs ante expresiones faciales

neutras y de felicidad.

P450:

$\downarrow$ amplitud en BDs y BDs codependientes que en Cs ante expresiones faciales

de felicidad.

Historia familiar de alcoholismo modula relación P450/BD.

P1, N2, P3b: $\uparrow$ latencia en BDs

No diferencias en amplitud.
Enlentecimiento en el desarrollo de sistemas cerebrales inhibitorios en BDs con policonsumo e historia familiar de alcoholismo.
N2: $\uparrow$ amplitud ante estímulos relevantes en BDs que en Cs.

P3: Igual amplitud ante estímulos relevantes e irrelevantes en BDs.

No efectos grupo $x$ sexo.
Aumento de latencias en BDs: enlentecimiento de la actividad cerebral similar a la de alcohólicos. Posible marcador electrofisiológico de BD.
Componente positivo tardío (LPC): $\downarrow$ amplitud, asociada con hipoactivación del CPF anterior dcho., ante estímulos relevantes en BDs.

No efectos grupo $x$ sexo.
Los BDs requieren mayores niveles de esfuerzo atencional y presentan deficiencias en la discriminación electrofisiológica entre estímulos relevantes e irrelevantes. $\uparrow$ de la fuerza espectral en las bandas delta $(0-4 \mathrm{~Hz})$ y beta rápido $(20-30 \mathrm{~Hz})$ en los BDs intensivos en comparación con los Cs y los BDs moderados.
Los BDs presentan alteraciones funcionales en el CPF relacionadas con los procesos de memoria de trabajo visual.
P1: $\uparrow$ amplitud en BDs ante estímulos relevantes relacionados con el alcohol (neutros y emocionales) en comparación con los no relacionados. N2b y P3: no diferencias.
Los BDs presentan signos de procesamiento prioritario (mayor asignación de recursos atencionales) de estímulos relacionados con el alcohol.
BDs intensivos presentaban $\uparrow$ latencia y/o $\downarrow$ amplitud en diversos componentes implicados en las distintas etapas del procesamiento cognitivo (P100, N100, N170, P2, N2b, P3a, P3b).

BDs moderados presentaban $\downarrow$ amplitud en los mismos componentes pero $\uparrow$ latencia sólo en P3b.

CDs no presentaron diferencias relevantes con Cs.

No efectos grupo $x$ sexo.
El BD afecta a los procesos cognitivos de nivel básico (percepción y atención y superior (decisión) y tiene un efecto más dañino que el consumo regular de alcohol per se.
P3b: $\uparrow$ amplitud en BDs que en Cs.

No diferencias en latencia.

N2: no diferencias en latencia ni amplitud.

No efectos grupo $x$ sexo.
Los BDs presentan anomalías (incrementado reclutamiento neural) en los procesos que median la atención visual. 


\begin{tabular}{|c|c|c|c|c|c|}
\hline Estudio & Grupos & Edad & Criterio BD & Objetivos & Tarea \\
\hline $\begin{array}{l}\text { López- } \\
\text { Caneda et } \\
\text { al., } 2012\end{array}$ & $\begin{array}{l}\cdot 25 \mathrm{Cs}\left(11 \delta^{\lambda}, 14 \text { 이 }\right. \\
\cdot 23 \mathrm{BDs}(13 \hat{\jmath}, 10+)\end{array}$ & $\begin{array}{l}\text { Inicio: } \\
\text { Rango edad: } 18-19 \\
\text { Cs: } 18,6 \pm 0,5 \\
\text { BDs: } 18,8 \pm 0,5 \\
\text { Seguimiento: } \\
\text { Rango edad: } 20-21 \\
\text { Cs: } 20,3 \pm 0,5 \\
\text { BDs: } 20,7 \pm 0,6\end{array}$ & $\begin{array}{l}\geq 6 \text { UBEs/ocasión } \\
\geq 1 \mathrm{vez} / \mathrm{mes} \\
\geq 3 \mathrm{bbs} / \mathrm{h} \\
0 \text { bien: } \\
\geq 6 \mathrm{UBEs} / \text { ocasión } \\
\geq 1 \mathrm{vez} / \text { semana }\end{array}$ & $\begin{array}{l}\text { Estudiar la actividad cerebral } \\
\text { durante la ejecución y la } \\
\text { inhibición de respuesta en } \\
\text { jóvenes BDs que mantienen } \\
\text { el patrón durante al menos } \\
2 \text { años. }\end{array}$ & - Tarea Go/NoGo \\
\hline $\begin{array}{l}\text { Petit et al., } \\
2013\end{array}$ & 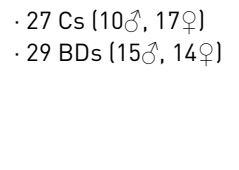 & 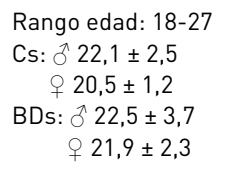 & $\begin{array}{l}\geq 6 \text { UBEs/ocasión } \\
\geq 3-4 \mathrm{vez} / \text { semana } \\
\geq 3 \mathrm{bbs} / \mathrm{h}\end{array}$ & $\begin{array}{l}\text { Analizar si los BDs presentan } \\
\text { aumentada reactividad } \\
\text { electrofisiológica ante } \\
\text { señales relacionadas con el } \\
\text { alcohol. }\end{array}$ & $\begin{array}{l}\text { - Tarea oddball visual con/sin } \\
\text { estímulos relacionados con } \\
\text { el alcohol }\end{array}$ \\
\hline $\begin{array}{l}\text { López- } \\
\text { Caneda et } \\
\text { al., } 2013\end{array}$ & $\begin{array}{l}\cdot 31 \mathrm{Cs}(15 \hat{0}, 16 \text { 아 }) \\
\cdot 26 \mathrm{BDs}(15 \hat{0}, 11 \text { 우 }\end{array}$ & $\begin{array}{l}\text { Inicio: } \\
\text { Rango edad: } 18-19 \\
\text { Cs: } 18,5 \pm 0,5 \\
\text { BDs: } 18,8 \pm 0,5 \\
\text { Seguimiento: } \\
\text { Rango edad: } 20-21 \\
\text { Cs: } 20,4 \pm 0,6 \\
\text { BDs: } 20,8 \pm 0,6\end{array}$ & $\begin{array}{l}\geq 6 \text { UBEs/ocasión } \\
\geq 1 \mathrm{vez} / \mathrm{mes} \\
\geq 3 \mathrm{bbs} / \mathrm{h} \\
0 \text { bien: } \\
\geq 6 \text { UBEs/ocasión } \\
\geq 1 \mathrm{vez} / \text { semana }\end{array}$ & $\begin{array}{l}\text { Estudiar la actividad cerebral } \\
\text { implicada en los procesos de } \\
\text { atención visual en jóvenes } \\
\text { BDs que mantienen este } \\
\text { patrón durante al menos } 2 \\
\text { años. }\end{array}$ & - Tarea oddball visual \\
\hline $\begin{array}{l}\text { Smith y } \\
\text { Mattick, } \\
2013\end{array}$ & $\begin{array}{l}\cdot 17 \mathrm{Cs}+ \\
\cdot 13 \mathrm{BDs} \text { ㅇ }\end{array}$ & $\begin{array}{l}\text { Rango edad: } 18-21 \\
\text { Cs: } 20,1 \pm 1,2 \\
\text { BDs: } 20,0 \pm 1,2\end{array}$ & $\begin{array}{l}\geq 4 \text { UBEs/ocasión } \\
\geq 1 \mathrm{vez} / \mathrm{mes}\end{array}$ & $\begin{array}{l}\text { Determinar si mujeres } \\
\text { jóvenes con BD presentan } \\
\text { déficits conductuales y/o } \\
\text { electrofisiológicos en el } \\
\text { control inhibitorio o en el } \\
\text { control/supervisión de la } \\
\text { ejecución. }\end{array}$ & - Tarea stop-signal \\
\hline $\begin{array}{l}\text { Watson et } \\
\text { al., } 2014\end{array}$ & $\begin{array}{l}\cdot 26 \mathrm{Cs}^{*}(13 \hat{\delta}, 139) \\
\cdot 16 \mathrm{BDs}^{*}(7 \hat{\jmath}, 9+)\end{array}$ & 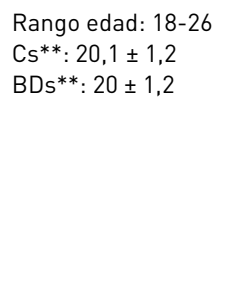 & $\begin{array}{l}\geq 5 / 4(\widehat{\delta} / \text { o }) \text { bbs en la misma } \\
\text { ocasión } \\
\geq 2 \text { veces últimos } 6 \text { meses } \\
\text { (historia familiar de alcoholismo } \\
\text { no evaluada) }\end{array}$ & $\begin{array}{l}\text { Determinar los } \\
\text { correlatos psicológicos y } \\
\text { neurofisiológicos de los } \\
\text { jóvenes BDs. } \\
\text { Adicionalmente, explorar } \\
\text { estos correlatos en los } \\
\text { consumidores de alcohol } \\
\text { mezclado con bebidas } \\
\text { cafeinadas. }\end{array}$ & $\begin{array}{l}\text { - Tarea oddball } \\
\text { - Tarea Go/NoGo }\end{array}$ \\
\hline
\end{tabular}

Nota. AUDIT: alcohol use disorders identification test; bbs: bebidas alcohólicas; BDs: binge drinkers; Cs: controles; CDs: consumidores diarios; CFI: córtex frontal inferior; CPF: córtex prefrontal; n.d.: no disponible; EEG: electroencefalograma; UBEs: unidades de bebida estándar. ${ }^{*}$ Número de sujetos incluidos en

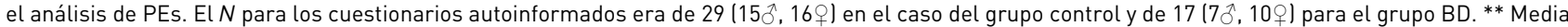
realizada incluyendo sujetos con historia personal de consumo de otras drogas distintas del alcohol, el tabaco y el cannabis; total Cs $=33$ (6 sujetos con historia personal otras drogas), total $\mathrm{BDs}=27$ sujetos (10 sujetos con historia personal otras drogas). 


\section{Principales Resultados}

P3-Go: $\uparrow$ amplitud en BDs que en Cs durante la ejecución de respuesta lal inicio y en el seguimiento).

P3-NoGo: $\uparrow$ amplitud en BDs asociada a hiperactivación del CFI dcho. durante

la inhibición de respuesta en el seguimiento.

N2: no diferencias en latencia ni amplitud.

No efectos grupo $x$ sexo.

\section{Conclusiones}

Los BDs presentan anomalías en la actividad cerebral relacionada con la ejecución y la inhibición de respuesta (neurocompensación dirigida a una ejecución eficiente de la tarea), lo cual puede constituir un antecedente neural de dificultades en el control de impulsos.
P3: $\uparrow$ amplitud en BDs ante estímulos relacionados con el alcohol en comparación con estímulos no alcohólicos. Análisis post-hoc revelaron que esta diferencia era significativa solamente en varones.

No diferencias en latencia.
BDs (especialmente varones) presentan incrementada reactividad electrofisiológica ante estímulos de tipo alcohólico, sugiriendo un mayor riesgo a desarrollar abuso de alcohol.
P3b: $\uparrow$ amplitud en BDs que en Cs al inicio del estudio, diferencia que era más pronunciada en el seguimiento.

Correlación negativa entre amplitud de P3b y edad de inicio de consumo de alcohol.

Correlación positiva entre amplitud de P3b y cantidad e intensidad de

consumo de alcohol.

No diferencias en latencia.

No efectos grupo $x$ sexo.
BDs presentan un reclutamiento adicional de recursos neurales relacionados con la atención y la memoria de trabajo, los cuales aumentan tras 2 años de mantenimiento del patrón BD. $\uparrow$ TR para inhibir la respuesta en BDs que en Cs.

P3: $\uparrow$ diferencia de amplitud ante inhibición correcta vs fallida en BDs que en Cs.

Esta diferencia entre inhibición correcta y fallida correlacionaba con la puntuación AUDIT.

Negatividad relacionada con el error (ERN): $\downarrow$ amplitud en BDs que en Cs.
Mujeres BDs necesitan más tiempo y un mayor esfuerzo cognitivo para inhibir correctamente la respuesta y presentan déficits en el control de la ejecución. $\uparrow$ amplitud P3a y P3b en BDs en tarea oddball.

Tendencia a mayor P3a (P3-NoGo) en BDs en tarea Go/NoGo.

Los consumidores de alcohol mezclado con bebidas cafeinadas presentaban

$\downarrow$ P3a en la tarea oddball.

No diferencias en P2 ni N2 entre grupos en ninguna de las dos tareas.
El BD está asociado con alteraciones (incrementada amplitud) en los componentes P3a y P3b de los PEs. 
Con respecto a la memoria declarativa episódica verbal, se ha utilizado el recuerdo de historias, en dos estudios del mismo grupo, y el aprendizaje de lista de palabras, en siete estudios de cuatro grupos de investigación. Mediante el paradigma de recuerdo de historias (que implica fundamentalmente actividad hipocampal), los resultados muestran menor capacidad de retención del contenido de las historias -tanto inmediata como demorada- en los jóvenes BDs en comparación con los consumidores ocasionales (Parada et al., 2011b). Estas diferencias se mantienen a los dos años de seguimiento entre los participantes que persisten en el patrón de consumo $\mathrm{BD}$, pero no entre quienes lo abandonan, lo que apoya la hipótesis de vulnerabilidad hipocampal a los efectos neurotóxicos del alcohol en adolescentes y constituye, a su vez, el primer índice de recuperación de una función cognitiva tras el abandono del patrón BD (Mota et al., 2013).

En el paradigma de aprendizaje de lista de palabras, sin embargo, las diferencias en las tareas empleadas en los diferentes trabajos parecen haber modulado los resultados. Así, los estudios que han empleado listas de palabras no relacionadas -el Test de Aprendizaje Auditivo Verbal de Rey (RAVLT) y una tarea de recuerdo demorado de palabras- no apuntaron diferencias en el recuerdo (Hartley et al., 2004; Mota et al., 2013; Parada et al., 2011b). Sin embargo, en tres de los cuatro estudios que usaron pruebas que implicaban palabras semánticamente relacionadas -el Test de Aprendizaje Verbal España-Complutense (TAVEC) y el California Verbal Learning Test (CVLT)- se observó menor rendimiento en los jóvenes BDs que en sus pares control (García-Moreno, Expósito, Sanhueza y Angulo, 2008; García-Moreno, Expósito, Sanhueza y Gil, 2009; Sneider, Cohen-Gilbert, Crowley, Paul y Silveri, 2013). En el trabajo de Sanhueza y colaboradores (2011), no obstante, no se encontraron diferencias entre los jóvenes BDs, los consumidores ocasionales y los abstemios en el recuerdo de palabras durante la ejecución del TAVEC.

La falta de consistencia entre estos estudios sobre memoria declarativa verbal podría estar relacionada, además de con las diferencias metodológicas, con la adopción, por parte de los jóvenes BDs, de estrategias alternativas de organización de la información, posibilitadas por tareas de aprendizaje de lista de palabras no relacionadas, con el objeto de compensar posibles limitaciones de almacenamiento y recuperación de la información verbal. Esta interpretación compensatoria es congruente con los resultados observados en los estudios neurofuncionales (López-Caneda et al., 2012; Schweinsburg, Schweinsburg, Nagel, Eyler y Tapert, 2011) (ver apartado siguiente).

Los siete trabajos disponibles en la literatura sobre la memoria episódica para material visual en los BDs, desarrollados por cuatro grupos de investigación, han empleado tareas de recuerdo libre y de pares asociados con estímulos tanto concretos como abstractos. Entre estos paradig- mas, los únicos que apuntan menor desempeño entre los jóvenes BDs son la tarea de aprendizaje de pares asociados de figuras abstractas (Paired Associates Learning, de la batería Cambridge Automated Neuropsychological Assessement Battery [CANTAB]) (Scaife y Duka, 2009) y el recuerdo de objetos comunes dibujados (Hartley et al., 2004). Las demás tareas no han identificado diferencias de rendimiento entre los jóvenes BDs y su grupo de comparación (García-Moreno et al., 2009; Mota et al., 2013; Parada et al., 2011b; Sanhueza et al., 2011; Sneider et al., 2013). Esta inconsistencia puede responder, de nuevo, a las diferencias en cuanto a nivel de dificultad, contenido estimular y grado de asociación semántica de los estímulos, y pone de manifiesto la necesidad de estudios de réplica para la contrastación de resultados.

Por último, un estudio ha informado de diferencias de rendimiento entre los grupos control y $\mathrm{BD}$ en una tarea que valoraba la memoria prospectiva (Heffernan, Clark, Bartholomew, Ling y Stephens, 2010). Pese a que no relatan más lapsus de memoria prospectiva que los jóvenes con bajo consumo de alcohol, los jóvenes BDs tienen más dificultades para recordar combinaciones localización-acción por medio del Prospective Remembering Video Procedure, una tarea que evalúa la memoria prospectiva diaria. Además, cuanto mayor es la cantidad de alcohol consumida semanalmente, menor es el rendimiento en la tarea, lo que sugiere que las alteraciones en la memoria prospectiva están relacionadas con el número de bebidas consumidas en los BDs (Heffernan et al., 2010).

Funciones Ejecutivas. Las funciones ejecutivas comprenden distintos procesos de manejo y organización de la información orientados a la consecución de un objetivo (Fuster, 2008). Esta habilidad neuropsicológica es la de mayor complejidad y la que ha despertado mayor interés entre los grupos de investigación. Trece de los 15 trabajos neuropsicológicos revisados analizaron el desempeño ejecutivo en los jóvenes con consumo intensivo de alcohol y todos ellos identificaron diferencias de rendimiento en alguna de las funciones estudiadas.

La memoria de trabajo verbal ha sido analizada en cinco trabajos diferentes por dos grupos españoles, a través de la tarea Dígitos y de Recitado de series. El grupo de García-Moreno y colaboradores encontró que los jóvenes BDs presentaban desempeño inferior a los controles en la puntuación total de Dígitos, una tarea que implica la repetición de las series numéricas en orden directo e inverso (García-Moreno et al., 2008; García-Moreno et al., 2009; Sanhueza et al., 2011), así como en el Recitado de series, un test que requiere realizar operaciones mentales sencillas durante 30 segundos (García Moreno et al., 2008). El grupo de Cadaveira y colaboradores ha constatado menor amplitud de retención y manipulación de dígitos en orden inverso entre los varones BD (Parada et al., 2012), diferencias que desaparecen tras dos años de seguimiento independientemente de la tra- 
yectoria del patrón de consumo (Mota et al., 2013). Este patrón, caracterizado por diferencias ligadas al sexo y mejora del rendimiento incluso con el mantenimiento del patrón de consumo, parece ser indicativo de una posible demora madurativa en los procesos relacionados con la memoria de trabajo más que una afectación ligada a la neurotoxicidad del patrón de consumo.

En estos cinco estudios, sumados a otros cinco trabajos, también se ha valorado la memoria de trabajo visoespacial en los BDs. Al emplear el paradigma de los Cubos de Corsi se replican parcialmente los resultados observados en las pruebas de memoria de trabajo verbal. Así, mientras que los jóvenes BDs presentan menos aciertos que los controles (García-Moreno et al., 2008; García-Moreno et al., 2009) excepto en el tercer trabajo del mismo grupo, que no ha encontrado diferencias (Sanhueza et al., 2011)-, estos sujetos con consumo intensivo de alcohol mantienen la misma amplitud de retención y manipulación de unidades de localización (García-Moreno et al., 2008; García-Moreno et al., 2009; Parada et al., 2012; Mota et al., 2013). Mediante el uso de la tarea Spatial Working Memory del CANTAB, dos trabajos apuntan a una mayor frecuencia de errores en las mujeres BD en comparación con las mujeres control (Scaife y Duka, 2009; Townshend y Duka, 2005), mientras un estudio no encuentra diferencias entre los grupos (Hartley et al., 2004).

Por último, entre los estudios que evalúan la memoria de trabajo, algunos han utilizado tareas de búsqueda auto-ordenada, como el Self-Ordered Pointing Task (SOPT), que requiere un alto grado de control cognitivo. En esta tarea, si bien los jóvenes BDs no presentaban diferencias en la cantidad de respuestas correctas (Johnson et al., 2008; Xiao et al., 2009), sí cometían mayor número de errores perseverativos (Parada et al., 2012; Mota et al., 2013), lo que parece reflejar dificultades en la supervisión de las estrategias utilizadas para resolver la tarea de forma eficiente.

Las habilidades de formación de conceptos y de flexibilidad cognitiva han sido exploradas por cuatro grupos de investigación distintos mediante el Intra-Extra Dimensional Set Shift (IED, del CANTAB) y el Wisconsin Card Sorting Task (WCST). En general, los jóvenes BDs no presentan dificultades en el aprendizaje de reglas en estas tareas y su habilidad de formación de concepto es similar a la de sus pares control (Hartley et al., 2004; Parada et al., 2012). Respecto a la flexibilidad cognitiva, esto es, la disposición o rigidez para el cambio en las estrategias cognitivas en respuesta a los cambios que se producen en las contingencias ambientales, los estudios indican desempeño similar entre los BDs y sus pares control (Mullan et al., 2011; Parada et al., 2012). Sin embargo, al considerar las diferencias sexuales, los trabajos apuntan hacia un efecto diferenciado del patrón BD en función del sexo. Así, mientras que los varones BDs se adaptan más fácilmente a cambios entre las dimensiones relevantes, ya que presentan menos errores en las etapas de cambio extradimensional (Hartley et al., 2004), las mujeres BDs presentan dificultades en la adquisición de nuevas reglas dentro de una misma dimensión, pues son más resistentes al cambio en el set de respuesta intradimensional (Scaife y Duka, 2009).

La capacidad de planificación ha sido estudiada por cuatro grupos de investigación a través de distintas tareas: Stockings of Cambridge (CANTAB), Torre de Hanoi, Mapa del Zoo y Búsqueda de Llaves. Los trabajos son concluyentes sobre el desempeño de los BDs, que se muestra similar al de los controles en todas estas tareas (García-Moreno et al., 2008; García-Moreno et al., 2009; Hartley et al., 2004; Mota et al., 2013; Parada et al., 2012; Sanhueza et al., 2011). Sin embargo, algunas diferencias, como mayor tiempo de planificación (Hartley et al., 2004) y menor tiempo total en la ejecución de la tarea (Mullan et al., 2011), destacan la posibilidad de subprocesos cognitivos diferenciados entre los grupos de comparación (controles y consumidores moderados de alcohol) y el grupo BD, si bien el rendimiento en las tareas de planificación no llega a verse afectado en los BDs.

En cuanto a los procesos de control inhibitorio, cuatro estudios de dos grupos de investigación diferentes han valorado esta función cognitiva por medio del test de Stroop, una tarea que permite estudiar la capacidad de controlar la interferencia automática. En tres de ellos se ha observado que los jóvenes con un patrón BD presentan un rendimiento más bajo que los consumidores ligeros (García-Moreno et al. 2008; García-Moreno et al. 2009; Sanhueza et al., 2011). Paradójicamente, el cuarto estudio ha encontrado mayor control inhibitorio entre consumidores moderados que entre los controles. Los autores interpretaron estos resultados como el reflejo del mayor esfuerzo que les supone a los consumidores moderados, en comparación con los jóvenes abstemios, inhibir o evitar el consumo intensivo de alcohol, sugiriendo así que el control inhibitorio puede ser fortalecido con la práctica (Mullan, Allom y Pack, 2011).

En tareas de tiempo de reacción simple y de elección ( $R e^{-}$ action Time y Match to Sample Visual Search, del CANTAB) se ha constatado que los BDs son más rápidos al elegir y ejecutar los movimientos de elección que los sujetos control, lo que ha sido interpretado como un índice de mayor impulsividad motora (Scaife y Duka, 2009; Townshend y Duka, 2005).

En cuanto a la toma de decisiones, analizada en cinco estudios de tres laboratorios diferentes y siempre a través del Iowa Gambling Task (IGT), se ha observado que los adolescentes con un patrón $\mathrm{BD}$, de forma análoga a las personas con abuso o dependencia de sustancias (Verdejo-García y Bechara, 2009), padecen cierta hipersensibilidad al refuerzo inmediato, ya que presentan preferencia por las recompensas positivas inmediatas, aún cuando esta elección resulta perjudicial a largo plazo (Johnson et al., 2008; Mullan et al., 2011; Xiao et al., 2009). Además, entre los BDs, aquellos que presentan un patrón más intenso de consumo de alcohol seleccionan menos opciones ventajosas que los consumidores ocasionales (Goudriaan, Grekin y Sher, 2007). 
En conjunto, el BD parece afectar de modo diferencial a las funciones mnésicas y ejecutivas dependientes de las regiones témporo-mesial y prefrontal. Además, este patrón de consumo se asocia con anomalías en procesos específicos dentro de las distintas funciones cognitivas. Todo ello pone de manifiesto la necesidad de administrar pruebas neuropsicológicas que permitan la diferenciación de los procesos cognitivos, así como la consideración de las diferencias sexuales, para la valoración en profundidad del desempeño en cada función cognitiva. Asimismo, estos hallazgos, aunque todavía preliminares, sugieren que la presencia de un patrón $\mathrm{BD}$ durante la adolescencia y la juventud está ligado, de forma general, a un rendimiento inferior en tareas que implican atención, aprendizaje, habilidades ejecutivas y toma de decisiones.

\section{Anomalías de tipo estructural y funcional ligadas al patrón binge drinking}

Impacto neuroestructural del BD. Hasta el momento, la mayoría de los estudios neuroestructurales han sido realizados en adolescentes y jóvenes con abuso o dependencia del alcohol (Welch, Carson y Lawrie, 2013), siendo todavía muy escasos los estudios en la población de adolescentes y jóvenes BDs. En la búsqueda realizada sólo se localizaron cinco estudios que hayan empleado neuroimagen estructural para examinar los cambios en la estructura cerebral asociados al BD en jóvenes y adolescentes sin diagnóstico de TCA (ver Tabla 2). Dos de ellos han empleado la técnica de imagen por tensor de difusión (ITD), para evaluar la integridad de la sustancia blanca, y los otros tres la técnica de IRM, para valorar la densidad de la sustancia gris cortical.

Los resultados obtenidos mediante ITD, procedentes de un único grupo de investigación, han mostrado que los adolescentes con un patrón BD presentan menor anisotropía fraccional (índice de complejidad estructural de los axones mielinizados) en diversos fascículos, incluyendo tractos de asociación (p.ej, los fascículos longitudinales superior e inferior), tractos de proyección (p.ej., la corona radiada) y tractos comisurales (p.ej., el cuerpo calloso) (Jacobus et al., 2009, McQueeny et al., 2009).

En cuanto a los estudios con IRM, los resultados indican la presencia de alteraciones estructurales asociadas al patrón BD en el cerebelo, el córtex frontal y el estriado ventral. Con respecto al cerebelo, se ha observado menor densidad tanto en la sustancia gris como en la sustancia blanca de ambos hemisferios en los jóvenes BDs en comparación con consumidores ocasionales (Lisdahl, Thayer, Squeglia, McQueeny y Tapert, 2013). En el córtex frontal las alteraciones observadas fueron distintas en hombres que en mujeres (Squeglia, Sorg et al., 2012). Así, mientras que las mujeres BDs presentaban mayor grosor cortical (mayor volumen de sustancia gris) en regiones frontales que las mujeres control, en el caso de los hombres se observó un patrón inverso de resultados (menor grosor cortical en los BDs). Estas diferencias entre sexos, según los autores, pueden ser debidas a que los hombres BDs eran significativamente mayores que sus pares control, por lo que su maduración cerebral (su poda sináptica) estaba más avanzada y, por lo tanto, su espesor cortical tendía a ser menor. Sobre la base de los resultados observados en las mujeres BDs, los autores propusieron que el consumo intensivo de alcohol durante la adolescencia estaba asociado con un retraso en el neurodesarrollo y, por lo tanto, con un mayor espesor cortical. En consonancia con estos resultados, un estudio reciente, procedente de otro grupo de investigación, ha observado mayor volumen de sustancia gris en el estriado ventral o núcleo accumbens (NAcc) en los jóvenes BDs que en los controles, lo que, según los autores, es indicativo de una relativa inmadurez neuroestructural en la población BD (Howell et al., 2013).

En definitiva, los escasos estudios de neuroimagen estructural disponibles sugieren que el patrón de consumo BD en la adolescencia se asocia con anomalías en la sustancia blanca (menor anisotropía fraccional) y en la sustancia gris (menor volumen cerebelar y mayor grosor cortical en regiones frontales). Aunque diversos trabajos con ITD han mostrado una correlación positiva entre integridad de las fibras de sustancia blanca y rendimiento neurocognitivo (Elofson, Gongvatana y Carey, 2013), se necesitan nuevos estudios para evaluar si las alteraciones en la sustancia blanca observadas en los jóvenes BDs podrían comprometer su funcionamiento cognitivo. En el caso de la sustancia gris, mientras que las alteraciones en el cerebelo parecen seguir el perfil apreciado en alcohólicos crónicos (Baker, Harding, Halliday, Krill y Harper, 1999; Harper, 2009), las afectaciones en el córtex frontal parecen específicas del patrón BD y son congruentes con un potencial retraso neuromadurativo en los jóvenes BDs.

Estudios de neuroimagen funcional. Junto a los estudios de neuroimagen estructural, en los últimos años han comenzado a estudiarse las consecuencias del BD por medio de la IRM funcional (IRMf). Estos trabajos, aunque todavía escasos, muestran que, aún en ausencia de diferencias en el nivel de ejecución de las tareas entre jóvenes BDs y jóvenes sin consumo intensivo de alcohol, el BD parece ejercer un impacto significativo sobre el funcionamiento cerebral en los jóvenes y adolescentes (Tabla 3). A partir de los términos de búsqueda ya mencionados en el apartado de método, se identificaron siete estudios (cinco de ellos del grupo de Tapert y cols.) que analizaban los efectos del BD por medio de la neuroimagen funcional.

Los primeros trabajos realizados mostraron diferencias en la actividad cerebral durante la codificación de pares de palabras (Schweinsburg, McQueeny, Nagel, Eyler y Tapert, 2010; Schweinsburg et al, 2011). Concretamente, los BDs presentaban mayor activación en regiones fronto-parietales así como hipoactivación en regiones del córtex occipital y 
el córtex cingulado, entre otras. Según los autores, estos resultados eran indicativos del empleo, por parte de los BDs, de sistemas de memoria alternativos durante el aprendizaje verbal, así como del mayor "esfuerzo neural" realizado para resolver la tarea eficientemente. Esta hipótesis resulta congruente con los resultados neuropsicológicos derivados de los test de aprendizaje verbal comentados en la sección anterior, los cuales sugerían la adopción por parte de los jóvenes BDs de estrategias alternativas de organización de la información verbal para compensar posibles limitaciones en el almacenamiento/recuperación de dicha información (Mota et al., 2013; Parada et al., 2011b).

En la misma línea, los resultados de otros dos estudios que evaluaron la memoria de trabajo visual en jóvenes y adolescentes sugieren que el BD conduce a cambios compensatorios en el funcionamiento cerebral -incremento de la actividad neural en regiones del córtex prefrontal, el cortex temporal, el hipocampo y el área motora suplementaria-, que permiten un rendimiento conductual equivalente al de los sujetos control (Campanella et al., 2013; Squeglia, Schweinsburg, Pulido y Tapert, 2011).

Tan sólo en uno de los estudios realizados hasta ahora se han observado diferencias a nivel conductual entre adolescentes con y sin un patrón BD (Xiao et al., 2013). En este trabajo, los adolescentes BDs presentaban una toma de decisiones menos eficiente, medida a través del IGT, que sujetos de la misma edad que no consumían alcohol. El hecho de que sólo se hayan encontrado diferencias conductuales en este estudio puede deberse a la mayor complejidad de esta tarea en comparación con las usadas en los restantes trabajos de neuroimagen, en muchos de los cuales es común llegar a un "efecto techo". Ello abre las puertas al empleo de tareas que entrañen una mayor complejidad cognitiva con el objeto de determinar la emergencia de alteraciones a nivel conductual en jóvenes BDs.

Finalmente, aunque el debate sobre si las anomalías en el funcionamiento cerebral observadas en los adolescentes BDs son resultado del consumo intensivo de alcohol o un factor de vulnerabilidad previo al comienzo del BD todavía persiste, recientes estudios de neuroimagen han aportado nueva luz a esta cuestión. Así, los únicos dos estudios longitudinales que abordan directamente este punto, autoría del grupo de Tapert y cols., apuntan en ambas direcciones: 1) a una actividad neural diferencial previa al inicio del consumo de alcohol (con menor actividad en regiones fronto-parietales en adolescentes BDs), lo cual podría constituir un factor de riesgo para el posterior consumo abusivo de sustancias; $y$ 2) a diferencias en el funcionamiento cerebral que emergen como consecuencia del patrón BD (con mayor actividad en regiones fronto-parietales en los BDs) (Squeglia, Pulido et al., 2012; Wetherill, Squeglia, Yang y Tapert, 2013).

Estudios psicofisiológicos. Se identificaron un total de 12 estudios psicofisiológicos, de cinco grupos de investigación diferentes (Tabla 4). En consonancia con los resultados derivados de los estudios de neuroimagen, los estudios psicofisiológicos mediante electroencefalografía (EEG) también han constatado la presencia de anomalías en el funcionamiento cerebral en jóvenes y adolescentes BDs. El único estudio que hasta la fecha ha estudiado la actividad cerebral basal en jóvenes con un patrón BD sin TCA observó que los BDs "intensivos" (más de 10 bebidas en un intervalo de dos horas en más de una ocasión en los últimos seis meses) presentaban mayor potencia en el espectro de las bandas de frecuencia delta $(0-4 \mathrm{~Hz})$ y beta rápido $(20-35 \mathrm{~Hz})$ del EEG en comparación con los bebedores ligeros y los BDs "moderados", lo que podría constituir, según los autores, un biomarcador de riesgo para el alcoholismo (Courtney y Polich, 2010).

Mediante la técnica de los potenciales relacionados con eventos o potenciales evocados (PEs), se ha apreciado que la actividad eléctrica cerebral asociada con diferentes funciones cognitivas está afectada en adolescentes y jóvenes con un patrón BD. Así, se ha visto que componentes de los PEs asociados a procesos perceptivos $(\mathrm{P} 1 / \mathrm{N} 1)$, atencionales (N2/P3), de memoria de trabajo (P3) o de control inhibitorio (P3-NoGo) presentan valores de amplitud y/o latencia anómalos en los jóvenes BDs en comparación con los controles (Crego et al., 2009, 2010; 2012; Ehlers et al., 2007; López-Caneda et al., 2012, 2013b; Maurage, Pesenti, Philippot, Joassin y Campanella, 2009; Maurage et al., 2012; Petit et al., 2012; Petit, Kornreich, Verbanck y Campanella, 2013; Smith y Mattick, 2013; Watson, Sweeney y Louis, 2014). No existe, sin embargo, unanimidad en torno a los resultados derivados de estos estudios. Así, mientras algunos informan de mayores latencias y/o menores amplitudes en los jóvenes BDs en comparación con los controles (Ehlers et al., 2007; Maurage et al., 2009; 2012), otros han observado un patrón diferente de resultados, con mayores amplitudes en algunos componentes y sin diferencias en los valores de latencia (Crego et al., 2009, 2012; López-Caneda et al., 2012, 2013b; Watson et al., 2014).

Aunque las múltiples diferencias entre los estudios (distintos paradigmas experimentales, diferente composición y tamaño de la muestra de BDs, etc.) dificultan su análisis conjunto, dos hipótesis diferentes, aunque no excluyentes entre sí, han emergido a partir de estos resultados. Por un lado, la hipótesis que sugiere que el $\mathrm{BD}$ y el alcoholismo son dos etapas del mismo fenómeno que conducen a déficits psicofisiológicos paralelos antes que a alteraciones independientes (Maurage et al., 2012); esto es, a un aumento en la latencia y a una reducción en la amplitud de los componentes de los PEs (véase Porjesz y Begleiter, 2003; Campanella et al., 2009 para una revisión de los efectos del alcoholismo sobre los PEs). Por otro, la hipótesis que plantea el BD como un fenómeno diferenciado del alcoholismo, con manifestaciones psicofisiológicas diferentes entre ambas formas de consumo de alcohol (Crego et al., 2012; López-Caneda et al., 2012); esto es, una reducción (déficit) en los valores de amplitud 
en los alcohólicos crónicos y un aumento (neurocompensación) en dichos valores en los sujetos con un patrón BD.

Ambas hipótesis podrían coexistir si consideramos que unas características de consumo más similares a las de los pacientes con abuso o dependencia del alcohol pueden conducir a las anomalías electrofisiológicas propias de estos pacientes (mayor latencia y menor amplitud de los PEs), mientras que un patrón más acorde con los criterios de cantidad, frecuencia e intensidad de consumo de alcohol que se han tomado como referencia para definir el BD (NIAAA, 2004; Wechsler, Lee, Juo y Lee, 2000) podría derivar en un mecanismo neurocompensatorio (aumentada amplitud de los PEs).

En este sentido, en los estudios que han observado patrones electrofisiológicos similares entre BDs y alcohólicos, los sujetos considerados BDs presentaban en realidad niveles de consumo similares o incluso superiores a los sujetos con diagnóstico de TCA de otros estudios con PEs -p.ej, 140 y 168 unidades de bebidas estándar (UBEs) al mes en los participantes definidos como BDs en los estudios de Maurage et al., 2009 y 2012, respectivamente, frente a 95 y 50 UBEs en sujetos diagnosticados con TCA evaluados por Fein y Andrew (2011) y Cuzen y cols. (2013), respectivamente. Así pues, es posible que el perfil electrofisiológico de estos jóvenes considerados BDs fuese relativamente equivalente al de los sujetos con abuso de alcohol a causa de las elevadas cantidades de alcohol consumido, muy superiores a las registradas en otros estudios (tanto electrofisiológicos como de neuroimagen) con jóvenes BDs (Crego et al., 2012; López-Caneda, Rodríguez Holguín, Corral, Doallo y Cadaveira, 2014; Schweinsburg et al., 2011; Wetherill et al., 2013). En estos últimos parece existir, a su vez, un paralelismo entre los resultados, encontrándose mediante ambas técnicas (IRMf y PEs) un incremento en la actividad neural en los BDs que permitiría inicialmente compensar el posible déficit subyacente.

Conjuntamente, estos resultados parecen apuntar hacia anomalías neurales -cuantificables mediante las técnicas electrofisiológicas y de neuroimagen- en los jóvenes y adolescentes BDs, que podrían ser compensadas por medio del reclutamiento de recursos neurales adicionales. Es posible sin embargo que, si este patrón de consumo intensivo se mantiene y/o se intensifica, el cerebro pueda no ser capaz de compensar el déficit subyacente y, consecuentemente, derive en un reducido funcionamiento neural. Será necesario llevar a cabo nuevos y más prolongados estudios de tipo longitudinal para comprobar estas hipótesis.

\section{Conclusiones}

El consumo intensivo de alcohol o BD constituye la forma de consumo problemático más común durante la adolescencia y juventud. Su alta prevalencia, junto con la evidencia derivada de los estudios con animales -que han mostrado la especial vulnerabilidad del cerebro adolescente a los efectos del alcohol-, ha alentado el interés en torno a las conse- cuencias neurocognitivas ligadas al BD en los jóvenes y adolescentes, lo que ha dado lugar a un creciente número de estudios publicados en la última década y, más concretamente, en el último lustro. Estas investigaciones asocian el consumo intensivo de alcohol en jóvenes con 1) alteraciones a nivel cognitivo, especialmente de las funciones mnésicas y ejecutivas dependientes de las regiones temporo-mesial y prefrontal; 2) desequilibrios neuroestructurales, con déficits en la sustancia blanca (en múltiples tractos de asociación, de proyección y comisurales) y en la sustancia gris cerebelar, así como retraso neuromadurativo (mayor espesor cortical) en regiones frontales y subcorticales y 3) anomalías neurofuncionales, con actividad compensatoria (hiperactivación) orientada probablemente a contrarrestar la menor actividad (hipoactivación) en otras regiones cerebrales con el fin de mantener un rendimiento conductual equivalente al de los sujetos sin consumo intensivo de alcohol.

Aunque todavía carecemos de estudios a largo plazo que hayan analizado si estas anomalías revierten tras el abandono de este patrón o se mantienen a lo largo del tiempo, de confirmarse los resultados expuestos en esta revisión, se hace necesaria una nueva y más detenida atención a esta problemática en los ámbitos educativos y de salud pública. La sensibilización respecto de los problemas derivados de esta forma de consumo de alcohol, junto con métodos de mejora/potenciación de aquellas habilidades cognitivas especialmente sensibles a los efectos del alcohol, podría traducirse en una reducción tanto de la prevalencia del patrón BD como del tiempo de mantenimiento de estos hábitos de consumo (véase, p.ej., los estudios de Houben, Wiers y Jansen, 2011; Houben, Nederkoorn, Wiers y Jansen, 2011; y de Bowley et al., 2013, relativos a los efectos del entrenamiento en el control inhibitorio y la memoria de trabajo sobre el consumo de alcohol). Todos estos resultados, sin embargo, son todavía incipientes y necesitan ser contrastados, preferentemente mediante estudios de seguimiento que comiencen a una edad temprana, previa al inicio del consumo de alcohol, de forma que permitan discernir con mayor claridad entre los factores de vulnerabilidad que pueden derivar en un consumo intensivo de alcohol y las consecuencias que puede acarrear este patrón de consumo.

\section{Reconocimientos}

Este trabajo ha sido financiado por el Plan Nacional de Formación de Profesorado Universitario (FPU) (referencia AP2008-03433) del Ministerio de Educación, por el programa nacional de bolsas posdoctorales de la Fundação para a Ciência e a Tecnologia (FCT) de Portugal (referencia SFRH/ BPD/91440/2012), por la Fundação de Amparo à Pesquisa do Estado do Rio de Janeiro (referencia 111.030/2013), por el Ministerio de Ciencia e Innovación, Plan Nacional de I+D (PSI2011-22575) y por el Ministerio de Sanidad y Política Social, Plan Nacional sobre Drogas (exp 2010/134). 


\section{Conflicto de intereses}

Los autores declaran no tener conflicto de intereses.

\section{Referencias}

Baker, K. G., Harding, A. J., Halliday, G. M., Kril, J. J., y Harper, C. G. (1999). Neuronal loss in functional zones of the cerebellum of chronic alcoholics with and without Wernicke's encephalopathy. Neuroscience, 91, 429-438. doi:10.1016/S0306-4522(98)90664-9.

Bava, S., y Tapert, S. F. (2010). Adolescent brain development and the risk for alcohol and other drug problems. Neuropsychology review, 20, 398-413. doi:10.1007/ s11065-010-9146-6.

Bechara, A., Damasio, A. R., Damasio, H., y Anderson, S. W. (1994). Insensitivity to future consequences following damage to human prefrontal cortex. Cognition, 50, 7-15.

Becker H. C. (1998). Kindling in alcohol withdrawal. Alcohol Health Res World, 22, 25-33.

Beirness, D. J., Foss, R. D., y Vogel-Sprott, M. (2004). Drinking on campus: self-reports and breath tests. Journal of Studies on Alcohol, 65, 600-604.

Blakemore, S. J. (2012). Imaging brain development: the adolescent brain. Neuroimage, 61, 397-406. doi:10.1016/j. neuroimage.2011.11.080.

Bowley, C., Faricy, C., Hegarty, B., Johnstone, S., Smith, J., Kelly, P., y Rushby, J. (2013). The effects of inhibitory control training on alcohol consumption, implicit alcohol-related cognitions and brain electrical activity. International Journal of Psychophysiology, 89, 342-348. doi:10.1016/j.ijpsycho.2013.04.011

Cadaveira, F. (2009). Alcohol y cerebro adolescente. Adicciones: Revista de socidrogalcohol, 21, 9-14.

Campanella, S., Petit, G., Maurage, P., Kornreich, C., Verbanck, P., y Noel, X. (2009). Chronic alcoholism: insights from neurophysiology. Clinical Neurophysiology, 39, 191-207. doi:10.1016/j.neucli.2009.08.002.

Campanella, S., Peigneux, P., Petit, G., Lallemand, F., Saeremans, M., Nöel, X., ... De Witte, P. (2013). Increased cortical activity in binge drinkers during working memory task: A preliminary assessment through a functional magnetic resonance imaging study. PLoS One 8, e62260. doi:10.1371/journal.pone.0062260.

Casey, B. J., Galvan, A., y Hare, T. A. (2005). Changes in cerebral functional organization during cognitive development. Current Opinion in Neurobiology, 15, 239-44. doi: 10.1016/j.conb.2005.03.012

Casey, B. J., Giedd, J. N., y Thomas, K. M. (2000). Structural and functional brain development and its relation to cognitive development. Biological Psychology, 54, 241-257. doi:10.1016/S0301-0511(00)00058-2.

Courtney, K. E., y Polich, J. (2010). Binge drinking effects on EEG in young adult humans. International journal of environmental research and public health, 7, 2325-2336. doi: $10.3390 /$ ijerph7052325

Courtney, K. E., y Polich, J. (2009). Binge drinking in young adults: Data, definitions, and determinants. Psychological Bulletin, 135, 142-156. doi: 10.1037/a0014414.

Crego, A., Cadaveira, F., Parada, M., Corral, M., Caamaño-Isorna, F., y Rodríguez Holguín, S. (2012). Increased amplitude of P3 event-related potential in young binge drinkers. Alcohol, 46, 415-425. doi:10.1016/j.alcohol.2011.10.002.

Crego, A., Rodríguez Holguín, S., Parada, M., Mota, N., Corral, M., y Cadaveira, F. (2009). Binge Drinking Affects Attentional and Visual Working Memory Processing in Young University Students. Alcoholism, Clinical and Experimental Research, 33, 1-10. doi:10.1111/j.15300277.2009.01025.x.

Crego, A., Rodríguez Holguín, S., Parada, M., Mota, N., Corral, M., y Cadaveira, F. (2010). Reduced anterior prefrontal cortex activation in young binge drinkers during a visual working memory task. Drug and Alcohol Dependence, 109, 45-56. doi:10.1016/j.drugalcdep.2009.11.020.

Crews, F. T., Braun, C. J., Hoplight, B., Switzer, R. C., y Knapp, D. J. (2000). Binge ethanol consumption causes differential brain damage in young adolescent rats compared with adult rats. Alcoholism: Clinical and Experimental Research, 24, 1712-1723. doi: 10.1111/j.1530-0277.2000.tb01973.x

Cuzen, N. L., Andrew, C., Thomas, K. G., Stein, D. J., y Fein, G. (2013). Absence of P300 reduction in South African treatment-Naïve adolescents with alcohol dependence. Alcoholism: Clinical and Experimental Research, 37, 4048. doi:10.1111/j.1530-0277.2012.01837.x.

Dahl, R.E. (2004). Adolescent brain development: a period of vulnerabilities and opportunities. Keynote address. Annals of the New York Academy of Sciences, 1021, 1-22. doi: 10.1196/annals.1308.001

Dubois, J., Dehaene-Lambertz, G., Perrin, M., Mangin, J. F., Cointepas, Y., Duchesnay, E., ... Hertz-Pannier, L. (2008). Asynchrony of the early maturation of white matter bundles in healthy infants: quantitative landmarks revealed noninvasively by diffusion tensor imaging. $\mathrm{Hu}$ man Brain Mapping, 29, 14-27. doi:10.1002/hbm.20363.

Duka, T., Gentry, J., Malcolm, R., Ripley, T.L., Borlikova, G., Stephens, D.N., ... y Crews, F.T. (2004). Consequences of multiple withdrawals from alcohol. Alcoholism: Clinical and Experimental Research, 28, 233-46. doi:10.1097/01. ALC.0000113780.41701.81.

Ehlers, C. L., Phillips, E., Finnerman, G.,Gilder, D., Lau, P., y Criado, J. (2007). P3 components and adolescent binge drinking in Southwest California Indians. Neurotoxicology and Teratology, 29, 153-163. doi:10.1016/j. ntt.2006.11.013.

Elofson, J., Gongvatana, W., y Carey, K. B. (2013). Alcohol Use and Cerebral White Matter Compromise in Adolescence. Addictive behaviors, 38, 2295-305. doi:10.1016/j. addbeh.2013.03.001. 
Eurobarometer (2010). EU citizens' attitudes towards alcohol. Special Eurobarometer 331 / Wave 72.3. Brussels: TNS Opinion \& Social at the request of Directorate General Health and Consumers and coordinated by the Directorate-General for Communication of European Commission.

Farke, W., y Anderson, P. (2007) Binge Drinking in Europe. Adicciones: Revista de socidrogalcohol, 19, 333-339.

Fein, G., y Andrew, C. (2011). Event-related potentials during visual target detection in treatment-naïve active alcoholics. Alcoholism: Clinical and Experimental Research, 35, 1171-1179. doi:10.1111/j.1530-0277.2011.01450.x.

Fuster, J. M. (2001) The prefrontal cortex-an update: time is of the essence. Neuron, 30, 319-33. doi:10.1016/S0896$6273(01) 00285-9$.

Fuster, J. M. (2002). Frontal lobe and cognitive development. Journal of Neurocytology, 31, 373-385. doi: 10.1023/A:1024190429920.

Fuster, J. M. (2008). The prefrontal cortex. London: Academic Press.

García-Moreno, L. M., Expósito J., Sanhueza, C., y Angulo, M. (2008). Actividad prefrontal y alcoholismo de fin de semana en jóvenes. Adicciones: Revista de socidrogalcohol, 20, 271-279.

García-Moreno, L. M., Expósito, J., Sanhueza, C., y Gil, S. (2009). Rendimiento cognitivo y consumo de alcohol durante los fines de semana en mujeres adolescentes. Revista Neuropsicología, Neuropsiquiatría y Neurociencias, 9, 75-91.

Giedd, J.N. (2004). Structural magnetic resonance imaging of the adolescent brain. Annals of the New York Academy of Sciences, 1021, 77-85. doi:10.1196/annals.1308.009.

Giedd, J. N., Blumenthal, J., Jeffries, N. O., Castellanos, F. X., Liu, H., Zijdenbos, A., ... Rapoport, J. L. (1999). Brain development during childhood and adolescence: a longitudinal MRI study. Nature Neuroscience, 2, 861-863. doi:10.1038/13158.

Giedd, J. N., Lalonde, F. M., Celano, M. J., White, S. L., Wallace, G. L., Lee, N. R., y Lenroot, R. K. (2009). Anatomical brain magnetic resonance imaging of typically developing children and adolescents. Journal of the American Academy of Child and Adolescent Psychiatry, 48, 465470. doi:10.1097/CHI.0b013e31819f2715.

Giedd, J. N., Snell, J. W., Lange, N., Rajapakse, J. C., Casey, B. J., Kozuch, P. L., ... Rapoport, J. L. (1996). Quantitative magnetic resonance imaging of human brain development: ages 4-18. Cerebral Cortex, 6, 551-560. doi:10.1093/ cercor/6.4.551.

Gill, J. S. (2002). Reported levels of alcohol consumption and binge drinking within the UK undergraduate student population over the last 25 years. Alcohol, 37, 109120. doi: 10.1093/alcalc/37.2.109.

Gogtay, N., Giedd, J. N., Lusk, L., Hayashi, K. M., Greenstein, D.,Vaituzis, A. C., ... Thompson, P. M. (2004). Dynamic mapping of human cortical development during childhood through early adulthood. Proceedings of the
National Academy of Sciences of the United States of America, 101, 8174-8179. doi:10.1073/pnas.0402680101.

Goslawski, M., Piano, M. R., Bian, J. T., Church, E., Szczurek, M., y Phillips, S. A. (2013). Binge drinking impairs vascular function in young adults. Journal of the American College of Cardiology, 62, 201-207. doi:10.1016/j. jacc.2013.03.049.

Goudriaan, A. E., Grekin, E. R., y Sher, K. J. (2007). Decision making and binge drinking: A longitudinal study. Alcoholism, Clinical and Experimental Research, 31, 928-938. doi:10.1111/j.1530-0277.2007.00378.x.

Guerri, C., y Pascual, M. (2010). Mechanisms involved in the neurotoxic, cognitive, and neurobehavioral effects of alcohol consumption during adolescence. Alcohol, 44, 15-26. doi:10.1016/j.alcohol.2009.10.003.

Ham, L. S., y Hope, D. A. (2003). College students and problematic drinking: a review of the literature. Clinical Psychology Review, 23, 719-759. doi:10.1016/S02727358(03)00071-0

Harper, C. (2009). The neuropathology of alcohol-related brain damage. Alcohol and Alcoholism, 44, 136-40. doi:10.1093/alcalc/agn102.

Hartley, D. E., Elsabagha, S., y File, S. E. (2004). Binge drinking and sex: effects on mood and cognitive function in healthy young volunteers. Pharmacology, Biochemistry and Behavior, 78, 611-619. doi:10.1016/j.pbb.2004.04.027.

Heffernan, T., Clark, R., Bartholomew, J., Ling, J., y Stephens, S. (2010). Does binge drinking in teenagers affect their everyday prospective memory? Drug and alcohol dependence, 109, 73-78. doi:10.1016/j.drugalcdep.2009.12.013.

Hermoye, L., Saint-Martin, C., Cosnard, G., Lee, S. K., Kim, J., Nassogne, M. C., ... Mori, S. (2006). Pediatric diffusion tensor imaging: normal database and observation of the white matter maturation in early childhood. Neuroimage, 29, 493-504. doi:10.1016/j.neuroimage.2005.08.017.

Hibell, B., Guttormsson, U., Ahlström, S., Balakireva, O., Bjarnason, T., Kokkevi, A., y Kraus, L. (2009). The ESPAD report 2007. Alcohol and other drug use among students in 35 european countries. Stockholm: The Swedish council for information on alcohol and other drugs (CAN) and the European Monitoring Centre for Drugs and Drug Addiction (EMCDDA).

Houben, K., Wiers, R. W., y Jansen, A. (2011). Getting a grip on drinking behavior: training working memory to reduce alcohol abuse. Psychological Science, 22, 968-975. doi: $10.1177 / 0956797611412392$.

Houben, K., Nederkoorn, C., Wiers, R. W., y Jansen, A. (2011). Resisting temptation: decreasing alcohol-related affect and drinking behavior by training response inhibition. Drug and Alcohol Dependence, 116, 132-136. doi:10.1016/j.drugalcdep.2010.12.011

Hooper, C. J., Luciana, M., Conklin, H. M., y Yarger, R. S. (2004). Adolescents' performance on the 
Iowa Gambling Task: implications for the development of decision making and ventromedial prefrontal cortex. Developmental psychology, 40, 1148-1158. doi:10.1037/0012-1649.40.6.1148.

Howell, N. A., Worbe, Y., Lange, I., Tait, R., Irvine, M., Banca, P., ... Voon, V. (2013). Increased ventral striatal volume in college-aged binge drinkers. PloS one, $8, e 74164$. doi:10.1371/journal.pone.0074164.

Huttenlocher, P. R., De Courten, C., Garey, L. J., y Van Der Loos, H. (1983). Synaptic development in human cerebral cortex. International Journal of Neurology, 16-17, 144-154.

Jacobus, J., McQueeny, T., Bava, S., Schweinsburg, B. C., Frank, L. R., Yang, T. T., y Tapert, S. F. (2009). White matter integrity in adolescents with histories of marijuana use and binge drinking. Neurotoxicology and Teratology, 31, 349-355. doi:10.1016/j.ntt.2009.07.006.

Jacobus, J., y Tapert, S. F. (2013). Neurotoxic effects of alcohol in adolescence. Annual review of clinical psychology, 9, 703-721. doi:10.1146/annurev-clinpsy-050212-185610.

Johnson, C. A., Xiao, L., Palmer, P., Sun, P., Wang, Q., Wei, Y., ... Bechara, A. (2008). Affective decision-making deficits, linked to a dysfunctional ventromedial prefrontal cortex, revealed in 10th grade Chinese adolescent binge drinkers. Neuropsychologia, 46, 714-726. doi:10.1016/j. neuropsychologia.2007.09.012.

Kraus, L., Baumeister, S. E., Pabst, A., y Orth, B. (2009). Association of average daily alcohol consumption, binge drinking and alcohol-related social problems: results from the German epidemiological surveys of substance abuse. Alcohol and Alcoholism, 44, 314-320. doi:10.1093/ alcalc/agn110.

Lange, J. E., Clapp, J. D., Turrisi, R., Reavy, R., Jaccard, J. . Johnson, M. B., ... Larimer, M. (2002). College binge drinking: what is it? Who does it? Alcoholism, Clinical and Experimental Research, 26, 723-730. doi: 10.1111/j.15300277.2002.tb02597.x

Lebel, C., y Beaulieu, C. (2011) Longitudinal development of human brain wiring continues from childhood into adulthood. The Journal of Neuroscience, 31, 10937-47. doi:10.1111/j.1530-0277.2002.tb02597.x.

Lisdahl, K. M., Thayer, R., Squeglia, L. M., McQueeny, T. M., y Tapert, S. F. (2013). Recent binge drinking predicts smaller cerebellar volumes in adolescents. Psychiatry Research: Neuroimaging, 211, 17-23. doi:10.1016/j. pscychresns.2012.07.009.

López-Caneda, E., Cadaveira, F., Crego, A., Gómez-Suárez, A., Corral, M., Parada, M., ... Rodríguez Holguín, S. (2012). Hyperactivation of right inferior frontal cortex in young binge drinkers during response inhibition: a follow-up study. Addiction, 107, 1796-1808. doi: 10.1111/j.1360-0443.2012.03908.x.

López-Caneda, E., Cadaveira, F., Crego, A., Doallo, S., Corral, M., Gómez-Suárez, A.F., y Rodríguez Holguín, S. (2013). Effects of a persistent binge drinking pattern of alcohol consumption in young people: a follow-up study using event-related potentials. Alcohol and Alcoholism 48, 464-471. doi:10.1093/alcalc/agt046.

López-Caneda, E., Rodríguez Holguín, S., Cadaveira, F., Corral, M., y Doallo, S. (2014). Impact of alcohol use on inhibitory control (and vice versa) during adolescence and young adulthood: a review. Alcohol and Alcoholism 49, 173-181. doi:10.1093/alcalc/agt168.

López-Caneda, E., Rodríguez Holguín, S., Corral, M., Doallo, S. y Cadaveira, F. (2014). Evolution of the binge drinking pattern in college students: Neurophysiological correlates. Alcohol, 48, 407-418. doi: 10.1016/j.alcohol.2014.01.009

Luna, B., y Sweeney, J.A. (2004). The emergence of collaborative brain function: FMRI studies of the development of response inhibition. Annals of the New York Academy of Sciences, 1021, 296-309. doi:10.1196/annals.1308.035.

Markwiese, B. J., Acheson, S. K., Levin, E. D., Wilson, W. A., y Swartzwelder, H. S. (1998). Differential effects of ethanol on memory in adolescent and adult rats. Alcoholism: Clinical and Experimental Research, 22, 416-421. doi:10.1111/j.1530-0277.1998.tb03668.x.

Maurage, P., Joassin, F., Speth, A., Modave, J., Philippot, P., y Campanella, S. (2012). Cerebral effects of binge drinking: respective influences of global alcohol intake and consumption pattern. Clinical Neurophysiology, 123, 892901. doi:10.1016/j.clinph.2011.09.018.

Maurage, P., Pesenti, M., Philippot, P., Joassin, F., y Campanella, S. (2009). Latent deleterious effects of binge drinking over a short period of time revealed only by electrophysiological measures. Journal of Psychiatry and Neuroscience, 34, 111-118.

McQueeny, T., Schweinsburg, B. C., Schweinsburg, A. D., Jacobus, J., Bava, S., Frank, L. R., y Tapert, S. F. (2009). Altered white matter integrity in adolescent binge drinkers. Alcoholism, Clinical and Experimental Research, 33, 1278-1285. doi:10.1111/j.1530-0277.2009.00953.x.

Mota, N., Álvarez-Gil, R., Corra,l M., Rodríguez Holguín, S., Parada, M., Crego, A.,... Cadaveira, F. (2010). Risky alcohol use and heavy episodic drinking among Spanish University students: a two-year follow-up. Gaceta Sanitaria, 24, 372-377. doi:10.1016/j.gaceta.2010.02.013.

Mota, N., Parada, M., Crego, A., Doallo, S., Caamaño-Isorna, F., Rodríguez Holguín, S., ... Corral, M. (2013). Binge drinking trajectory and neuropsychological functioning among university students: A longitudinal study. Drug and Alcohol Dependence, 133, 108-114. doi:10.1016/j. drugalcdep.2013.05.024.

Mullan, B., Wong, C., Allom, V., y Pack, S. L. (2011). The role of executive function in bridging the intention-behaviour gap for binge-drinking in university students. Addictive behaviors, 36, 1023-1026.

Naimi, T. S., y Brewer, R. D. (2005). "Binge" drinking and blood alcohol concentration. Journal of Studies on Alcohol, $66,438-440$. 
National Institute of Alcohol Abuse and Alcoholism (2004). NIAAA council approves definition of binge drinking. NIAAA Newsletter, 3, p. 3. Recuperado de http:/ / pubs.niaaa.nih.gov/publications/Newsletter/ winter2004/Newsletter_Number3.pdf

Observatorio Europeo de las Drogas y las Toxicomanías (OEDT) (2011). Situación y tendencias de los problemas de drogas en España. Informe 2011. Secretaría General de Política Social y Consumo, Delegación del Gobierno para el Plan Nacional sobre Drogas. Madrid: Ministerio de Sanidad, Política social e igualdad, Centro de Publicaciones.

Observatorio Europeo de las Drogas y las Toxicomanías (OEDT) (2012). Informe Nacional 2012 (datos del 2011) al OEDT por el Punto Focal Nacional Reitox (ESPAÑA). Evolución, Tendencias y Cuestiones Particulares. Secretaría General de Política Social y Consumo, Delegación del Gobierno para el Plan Nacional sobre Drogas. Madrid: Ministerio de Sanidad, Política social e igualdad, Centro de Publicaciones.

Observatorio Español de la Droga y las Toxicomanías (OEDT) (2013). Encuesta estatal sobre uso de drogas en enseñanzas secundarias (ESTUDES). Secretaría de Estado de Servicios Sociales e Igualdad, Delegación del Gobierno para el Plan Nacional sobre Drogas. Ministerio de Sanidad, Política social e igualdad. http://www.pnsd.msc.es/Categoria2/ observa/pdf/PresentESTUDES2012_2013.pdf

Østby, Y., Tamnes, C. K., Fjell, A. M., Westlye, L. T., Due-Tønnessen, P., y Walhovd, K. B. (2009). Heterogeneity in subcortical brain development: a structural magnetic resonance imaging study of brain maturation from 8 to 30 years. The Journal of Neuroscience, 29, 11772-11782. doi:10.1523/JNEUROSCI.1242-09.2009

Parada, M., Corral, M., Caamaño-Isorna, F., Mota, N., Crego, A., Rodríguez Holguín, S. y Cadaveira, F. (2011a). Definition of adolescent binge drinking. Adicciones, 23, 53-63. doi:10.1016/j.alcohol.2011.10.002

Parada, M., Corral, M., Caamaño-Isorna, F., Mota, N., Crego, A., Rodríguez Holguín, S., y Cadaveira, F. (2011b). Binge Drinking and Declarative Memory in University Students. Alcoholism: Clinical and Experimental Research, 35, 1475-1484. doi:10.1111/j.1530-0277.2011.01484.x.

Parada, M., Corral, M., Mota, N., Crego, A., Rodríquez Holguín, S. R., y Cadaveira, F. (2012). Executive functioning and alcohol binge drinking in university students. Addictive Behaviors, 37, 167-172. doi:10.1016/j.drugalcdep.2013.05.024.

Pascual, M., Pla, A., Miñarro, J., y Guerri, C. (2014). Neuroimmune activation and myelin changes in adolescent rats exposed to high-dose alcohol and associated cognitive dysfunction: a review with reference to human adolescent drinking. Alcohol and Alcoholism. Avance de publicación on-line. doi:10.1093/alcalc/agt164

Petit, G., Kornreich, C., Maurage, P., Noel, X., Letesson, C., Verbanck, P., y Campanella, S. (2012). Early attentional modulation by alcohol-related cues in young binge drinkers: an event-related potentials study. Clinical Neurophysiology, 123, 925-36. doi:10.1016/j. clinph.2011.10.042.

Petit, G., Kornreich, C., Verbanck, P., y Campanella, S. (2013). Gender differences in reactivity to alcohol cues in binge drinkers: A preliminary assessment of event-related potentials. Psychiatry Research 209, 494-503. doi:10.1016/j.psychres.2013.04.005.

Petit, G., Maurage, P., Kornreich, C., Verbanck, P., y Campanella, S. (2014). Binge Drinking in Adolescents: A Review of Neurophysiological and Neuroimaging Research. Alcohol and Alcoholism 49, 193-197.

Porjesz, B., y Begleiter, H. (2003). Alcoholism and human electrophysiology. Alcohol Research and Health, 27, 153-160.

Risher, M-L., Fleming, R. L., Boutros, N., Semenova, S., Wilson, W. A., Levin, E. D. ... Acheson, S. K. (2013). Longterm effects of chronic intermittent ethanol exposure in adolescent and adult rats: radial-arm maze performance and operant food reinforced responding. PLoS ONE 8, e62940. doi:10.1371/journal.pone.0062940.

Rehm, J., Rehm, M. X., Shield, K. D., Gmel, G., y Gual, A. (2013). Consumo de alcohol, dependencia alcohólica, trastornos relacionados con el alcohol en España. Impacto de los tratamientos de la dependencia alcohólica. Adicciones, 25, 11-18.

Sanhueza, C., García-Moreno, L. M., y Expósito, J. (2011). Weekend alcoholism in youth and neurocognitive aging. Psicothema, 23, 209-214.

Scaife, J. C., y Duka, T. (2009). Behavioural measures of frontal lobe function in a population of young social drinkers with binge drinking pattern. Pharmacology Biochemistry and Behavior, 93, 354-62. doi:10.1016/j. pbb.2009.05.015.

Schweinsburg, A. D., McQueeny, T., Nagel, B. J., Eyler, L. T., y Tapert, S. F. (2010). A preliminary study of functional magnetic resonance imaging response during verbal encoding among adolescent binge drinkers. Alcohol, 44, 111-117. doi:10.1016/j.alcohol.2009.09.032.

Schweinsburg, A. D., Schweinsburg, B. C., Nagel, B. J., Eyler, L. T., y Tapert, S. F. (2011). Neural correlates of verbal learning in adolescent alcohol and marijuana users. Addiction, 106, 564-573. doi:10.1111/j.13600443.2010.03197.x.

Shaw, P., Kabani, N. J., Lerch, J. P., Eckstrand, K., Lenroot, R., Gogtay, N., ... Wise, S. P. (2008). Neurodevelopmental trajectories of the human cerebral cortex. The Journal of Neuroscience, 28, 3586-3594. doi:10.1523/JNEUROSCI.5309-07.2008.

Sircar, R., y Sircar, D. (2005). Adolescent rats exposed to repeated ethanol treatment show lingering behavioral impairments. Alcoholism: Clinical and Experimental Research, 29, 1402-1410. doi:10.1097/01.alc.0000175012.77756.d9. Smith, J. L., y Mattick, R. P. (2013). Evidence of deficits in behavioural inhibition and performance monitoring in 
young female heavy drinkers. Drug and Alcohol Dependence, 133, 398-404. doi:10.1016/j.drugalcdep.2013.06.020.

Sneider, J. T., Cohen-Gilbert, J. E., Crowley, D. J., Paul, M.D., y Silveri, M.M. (2013). Differential effects of binge drinking on learning and memory in emerging adults. Journal of Addiction Research and Therapy, 7. doi:10.4172/2155-6105.S7-006.

Spear, L. (2000). The adolescent brain and age-related behavioral manifestations. Neuroscience Biobehavioral Reviews, 24, 417-463. doi:10.1016/S0149-7634(00)00014-2.

Spear, L. (2013). The teenage brain. Adolescents and alcohol. Current Directions in Psychological Science, 22, 152-7. doi:10.1177/0963721412472192.

Squeglia, L. M., Schweinsburg, A. D., Pulido, C., y Tapert, S. F. (2011). Adolescent binge drinking linked to abnormal spatial working memory brain activation: differential gender effects. Alcoholism: Clinical and Experimental Research, 35, 1831-1841. doi:10.1111/j.1530-0277.2011.01527.x.

Squeglia, L. M., Pulido, C., Wetherill, R. R., Jacobus, J., Brown, G. G., y Tapert, S. F. (2012b). Brain response to working memory over three years of adolescence: influence of initiating heavy drinking. Journal of Studies on Alcohol and Drugs 73, 749-760.

Squeglia, L. M., Sorg, S. F., Schweinsburg, A. D., Wetherill, R. R., Pulido, C., y Tapert, S. F. (2012). Binge drinking differentially affects adolescent male and female brain morphometry. Psychopharmacology, 220, 529-539. doi:10.1007/s00213-011-2500-4.

Substance Abuse and Mental Health Services Administration (2013). Results from the 2012 National Survey on Drug Use and Health: Summary of National Findings, NSDUH Series H-46, HHS Publication No. (SMA) 13-4795. Rockville, MD: Substance Abuse and Mental Health Services Administration.

Svensson, J., y Landberg, J. (2013). Is youth violence temporally related to alcohol? A time-series analysis of binge drinking, youth violence and total alcohol consumption in Sweden. Alcohol and Alcoholism, 48, 598-604. doi:10.1093/alcalc/agt035.

Tamm, L., Menon, V., y Reiss, A. L. (2002). Maturation of brain function associated with response inhibition. Journal of the American Academy of Child and Adolescent Psychiatry, 41, 1231-1238. doi:10.1097/00004583-200210000-00013.

Townshend, J. M., y Duka, T. (2005). Binge drinking, cognitive performance and mood in a population of young social drinkers. Alcoholism: Clinical and Expermiental Research, 29, 317-325. doi:10.1097/01.ALC.0000156453.05028.F5.

Valencia-Martín, J.L., Galan, I., y Rodríguez-Artalejo, F. (2008) The joint association of average volume of alcohol and binge drinking with hazardous driving behaviour and traffic crashes. Addiction, 103, 749-757. doi:10.1111/j.1360-0443.2008.02165.x.

Verdejo-García, A., y Bechara, A. (2009). A somatic marker theory of addiction. Neuropharmacology, 56, 48-62. doi:10.1016/j.neuropharm.2008.07.035.
Watson, T. D., Sweeney, J. F. y Louis, H. (2014). Neurocognitive, psychological and behavioral correlates of binge drinking and use of alcohol with caffeinated beverages in college-aged adults. The American Journal of Drug and Alcohol Abuse, 40, 58-66. doi:10.3109/00952990.2013.84 3005

Wechsler, H., Lee, J. E., Kuo, M., y Lee, H. (2000). College binge drinking in the 1990s: A continuing problem results of the Harvard School of Public Health 1999 College Alcohol Study. Journal of American College Health, 48, 199-210. doi:10.1080/07448480009599305.

Wechsler, H., Davenport, A., Dowdall, G., Moeykens, B., y Castillo, S. (1994). Health and behavioral consequences of binge drinking in college. A national survey of students at 140 campuses. Journal of the American Medical Association, 272, 1672-1677. doi:10.1001/ jama.1994.03520210056032.

Welch, K. A., Carson, A., y Lawrie, S. M. (2013). Brain structure in adolescents and young adults with alcohol problems: Systematic review of imaging studies. Alcohol and Alcoholism, 48, 433-444. doi:10.1093/alcalc/agt037.

Wetherill, R. R., Squeglia, L. M., Yang, T. T., y Tapert, S. F. (2013). A longitudinal examination of adolescent response inhibition: neural differences before and after the initiation of heavy drinking. Psychopharmacology, 230, 663-671. Avance de publicación on-line. doi:10.1007/ s00213-013-3198-2.

White, A. M., y Swartzwelder, H. S. (2004). Hippocampal function during adolescence: a unique target of ethanol effects. Annals of the New York Academy of Sciences, 1021, 206-220. doi:10.1196/annals.1308.026.

White, A. M., y Swartzwelder, H. S. (2005). Age-related effects of alcohol on memory and memory-related brain function in adolescents and adults. Recent Developments in Alcoholism, 17, 161-176. doi: 10.1007/0-306-48626-1_8.

Xiao, L., Bechara, A., Gong, Q., Huang, X., Li, X., Xue, G., ... Anderson, J. C. (2013). Abnormal affective decision making revealed in adolescent binge drinkers using a functional magnetic resonance imaging study. Psychology of Addictive Behaviors, 27, 443-454. doi:10.1037/a0027892.

Xiao, L., Bechara, A., Grenard, L. J., Stacy, W. A., Palmer, P., Wei, Y., ... Johnson, C. A. (2009). Affective decision-making predictive of Chinese adolescent drinking behaviors. Journal of the International Neuropsychological Society, 15, 547-557. doi:10.1017/S1355617709090808.

Yakovlev, P. A., y Lecours, I. R. (1967). The myelogenetic cycles of regional maturation of the brain. En A. Minkowski (Ed.), Regional development of the brain in early life (pp. 3-70). Oxford: Blackwell. 